\title{
RatSWD
}

\section{Regionale Bildungsstrukturen und Beschäftigung in Deutschland}

Mareike Tarazona

2007 


\section{Research Notes des Rates für Sozial- und Wirtschaftsdaten (RatSWD)}

In der Publikationsreihe RatSWD Research Notes erscheinen empirische Forschungsergebnisse, beruhend auf Daten, die über die durch den RatSWD empfohlene informationelle Infrastruktur zugänglich sind. Die Pre-Print-Reihe startete Ende 2007 unter dem Titel RatSWD Working Papers.

Arbeiten aus allen sozialwissenschaftlichen und wirtschaftswissenschaftlichen Disziplinen können in der Reihe erscheinen. Die Reihe RatSWD Research Notes bietet einen Einblick in die vielfältigen wissenschaftlichen Anwendungsmöglichkeiten empirischer Daten und Statistiken und richten sich somit an interessierte, empirisch arbeitende Wissenschaftler/innen ebenso wie an Vertreter/innen öffentlicher Einrichtungen der Datenerhebung und der Forschungsinfrastruktur.

Die RatSWD Research Notes sind eine Plattform für eine frühzeitige zentrale und weltweit sichtbare Veröffentlichung von auf empirischen Daten basierenden Forschungsergebnissen und konzeptionellen Ideen zur Gestaltung von Erhebungen. RatSWD Research Notes sind nicht-exklusiv, d. h. einer Veröffentlichung an anderen Orten steht nichts im Wege. Alle Arbeiten können und sollen auch in fachlich, institutionell und örtlich spezialisierten Reihen erscheinen. RatSWD Research Notes können nicht über den Buchhandel, sondern nur online über den RatSWD bezogen werden.

Um nicht deutsch sprechenden Leser/innen die Arbeit mit der neuen Reihe zu erleichtern, sind auf den englischen Internetseiten der RatSWD Research Notes nur die englischsprachigen Papers zu finden, auf den deutschen Seiten werden alle Nummern der Reihe chronologisch geordnet aufgelistet.

Die Inhalte der Ausgaben stellen ausdrücklich die Meinung der jeweiligen Autor/innen dar und nicht die des RatSWD.

Herausgeber der RatSWD Research Notes Reihe:

Vorsitzender des RatSWD (2007/08 Heike Solga, 2009 Gert G. Wagner)

Geschäftsführer des RatSWD (Denis Huschka) 


\title{
Regionale Bildungsstrukturen und Beschäftigung in Deutschland
}

Mareike Tarazona, Universität Flensburg

Expertise, Rat für Sozial- und Wirtschaftsdaten

Dezember 2006

\begin{abstract}
Da international vergleichende Studien trotz zunehmender Einigkeit über die Bedeutung von Humankapital für Wachstum und Beschäftigung mangelnde Vergleichbarkeit und Datenqualität von Bildungsvariablen zu inkonsistenten Ergebnissen kommen, wurde die Bedeutung von Humankapital mit Daten auf der Ebene der deutschen Raumordnungsregionen überprüft. Es konnte gezeigt werden, dass regionale Disparitäten für das Humankapital bestehen, die nicht im Zeitverlauf konvergieren. Die Altersstruktur der beschäftigten Bevölkerung ist hierfür nicht verantwortlich. Die Branchenzusammensetzung erweist sich als moderierende Variable für die Wirkung von Humankapital auf die Beschäftigungsentwicklung. Eine Wachstumsregression unterstreicht, dass dem Humankapital eine wichtige Rolle zukommt, was jedoch nicht für den Anteil von Beschäftigten mit Hochschulabschluss gilt. Signifikante Effekte haben aber die durchschnittlichen Jahre formaler Bildung der Beschäftigten und der Anteil von Abiturienten mit und ohne Berufsausbildung.
\end{abstract}

\section{Regionale statt internationaler Daten zur Humankapitalschätzung}

Wenngleich die Bedeutung des Humankapitals für das Wachstum einer Volkswirtschaft und dessen Beschäftigung immer weniger umstritten ist, lassen insbesondere die Probleme der Messbarkeit und der Vergleichbarkeit von Humankapitalvariablen Zweifel an der Bedeutung dieses Faktors zu. Die viel zitierte Studie von Levine und Renelt (1991) führt an, dass bei der Durchsicht einer Vielzahl von Publikationen über 50 verschiedene signifikante Variablen in Wachstumsregressionen zu finden sind, wobei die Bildungsvariablen ihre Signifikanz häufig bei gemeinsamer Betrachtung mit anderen makroökonomischen Variablen einbüßen. Im vorliegenden Beitrag wird daher versucht, einen alternativen Weg zur üblichen Verwendung internationaler Humankapitalvariablen zu bestreiten, indem Regionaldaten aus Deutschland eingesetzt werden.

Die meisten Studien verwenden internationale Daten zur Verteilung von Bildung, wie sie zum Beispiel von der UNESCO veröffentlicht werden. Diese Daten sind jedoch aufgrund einer Vielzahl methodischer Unsicherheiten der Kritik ausgesetzt. Unterschiede können durch unberücksichtigte Variablen zustande kommen, die teilweise nicht beobachtbar sind oder zu denen keine Daten zu Verfügung stehen. Institutionelle, kulturelle, historische oder politische Rahmenbedingungen sind vielfältig und im Hinblick auf die Variable ,Bildung' vermutlich besonders einflussreich.

Die Validität von Daten kann bei ländervergleichenden Studien als problematisch bezeichnet werden. De la Fuente und Doménech (2000) zeigen, dass die Datenqualität einen erheblichen Einfluss auf die Stärke und Signifikanz der Humankapitalvariablen in Wachstumsgleichungen hat. Die von innen gefundene Interkorrelation der HumankapitalDaten aus 23 OECD-Ländern aus zwei verschiedenen Studien beträgt nur .574 und ohne die vier Länder mit den niedrigsten Werten sogar Null $(r=.063)$. Um Ungenauigkeiten in den Daten zu vermeiden, werden zur Konstruktion der Bildungsvariablen zuweilen Datensätze miteinander kombiniert. 
Im Gegensatz zu internationalen Daten weisen regionale Variablen bedeutende Vorteile auf, da einige der beschriebenen Problematiken entfallen oder stark vermindert werden. (Dabei bedeutet "regional" hier und im Folgenden, dass verschiedene Regionen innerhalb eines einzigen Landes miteinander verglichen werden, sofern dies nicht anders ausgewiesen wird. ${ }^{1}$ ) So ist mit deutlich geringerem Einfluss unberücksichtigter Variablen auf die Ergebnisse von Zusammenhangsmaßen und Wachstumsgleichungen zu rechnen, da die Regionen in einem - trotz kleinerer Unterschiede und des Bildungsföderalismus institutionell einheitlichem Rahmen eingebettet sind. So werden Variationen vieler nichtbeobachtbarer Variablen reduziert. Dies trifft ebenfalls auf die Homogenität der Daten zu, die einer einheitlichen Registrierungssystematik unterliegen und vergleichbaren Quellen entstammen.

Da die Validität gerade in ärmeren Ländern und Ländern ohne mit Deutschland vergleichbarer Berichtspflicht besonders zweifelhaft ist, kann die Verwendung von Daten eines einzelnen OECD-Landes wie Deutschland zur Verbesserung der Validität beitragen. Zur Qualität der im vorliegenden Ansatz verwendeten Datensätze wird in Abschnitt 5 näher eingegangen.

\section{Theoretische Überlegungen zum Zusammenhang von Bildung und Beschäftigung: Wachstumstheorie und Regionalforschung}

Die Bedeutung von Bildung für Beschäftigung und technologische Entwicklung ist weder theoretisch noch empirisch ein neues Konzept. Darüber, auf welche Weise Bildung wirksam wird, besteht demnach weiterhin keine Einigung. Während die Wissensproduktion in neoklassischen Wachstumsmodellen exogen bestimmt ist, werden in Modellen der neuen Wachstumstheorie steigende Skalenerträge durch positive Externalitäten oder Spill-OverEffekte angenommen. Die jüngeren Ansätze der Wachstumstheorie platzieren also Humankapital als Motor endogenen Wachstums in ihre Modelle. Dem technischen Fortschritt wird in der Theorie ein erheblicher Beitrag zum Wirtschaftswachstum beigemessen. Die Rolle des Humankapitals wird in vielen Modellen als indirekter Einfluss verstanden, beispielsweise für die Absorption fremder Technologien und für die Anwendung von Technologien.

Empirische Überprüfungen der Modelle zeigen jedoch keinen konsistent positiven Einfluss des Humankapitals, während sich die Investitionsraten für physisches Kapital als der robusteste Einflussfaktor erweisen. Dabei wird ein erweitertes Solow-Modell häufig als Grundlage empirischer Studien herangezogen, in dem Investitionen, das Bevölkerungswachstum und Humankapital zur Erklärung von Wachstum beitragen. Besonders prominent ist die darauf basierende empirische Studie von Mankiw, Romer und Weil (1992).

In vielen Wachstumsmodellen wird von Konvergenz zwischen Ländern ausgegangen, die de facto nicht prinzipiell zu beobachten ist. Besser zu den stilisierten Fakten passt das Konzept der bedingten Konvergenz: Demzufolge gibt es Konvergenz, wenn andere Faktoren kontrolliert werden. Einer der wichtigen Faktoren ist dabei das Humankapital eines Landes. Weber (1998) kritisiert allerdings die Annahme der bedingten Konvergenz durch die Kontrolle von Humankapitalvariablen aufgrund dessen, dass die Variablen Humankapital und Schulbildung absolute Konvergenz aufweisen und daher nicht langfristige Disparitäten erklären können. Er zeigt dies für eine Reihe von Bildungsvariablen, unter anderem für die Unterschiede in den Bildungsniveaus von Männern und Frauen.

Im Vergleich zu international vergleichenden Untersuchungen gelten für regionalökonomische Fragestellungen besondere Bedingungen. In neueren Ansätzen der Regionalforschung werden jedoch Erkenntnisse der neuen Wachstumstheorie integriert

\footnotetext{
${ }^{1}$ In Abgrenzung zu anderen Studien, die sich auf regionale Unterschiede beziehen, die zwischen Ländern in verschiedenen Regionen der Welt und eines Kontinents existieren.
} 
(Bräuninger \& Niebuhr, 2005). So wird Agglomerationsprozessen zugeschrieben, dass sie Innovation begünstigen, und Annahmen zu Determinanten von Wachstum übernehmen, zu denen auch das Humankapital zählt.

Regionale Unterschiede in Niveau und Entwicklung von Beschäftigung und Arbeitslosigkeit innerhalb eines Staates wurden in jüngster Zeit für Länder fast aller Kontinente untersucht (z. B. (Brunelli, Lupi \& Ordine, 2001, Galiani et al., 2005, Ramakrishnan \& Cerisola, 2004, Walsh, 2003). In solchen Untersuchungen werden vergleichsweise selten Bildungsvariablen hinzugezogen. Elhorst (2003) machte deutlich, dass dies in weniger als einem Viertel der Veröffentlichungen der Fall ist. In sechs der neun von inm identifizierten Studien, welche das Humankapital einbeziehen, hatte die Bildungsvariable einen signifikant negativen Effekt auf die Arbeitslosigkeit. Eine Bildungsvariable wird in diesen Veröffentlichungen zumeist als Kontrollvariable einbezogen und die verwendeten Variablen unterscheiden sich. Dies liegt auch an dem Mangel verfügbarer Daten auf regionaler Ebene.

Feser (2003) macht auf Basis regionaler Zusammensetzungen eine empirische Analyse von Clustern, die verschiedene wissensbasierte Berufsgruppen zusammenfassen. Wie Feser hebt Elhorst (2003) die Bedeutung der Branchenzusammensetzung hervor, da diese oft als eine der Hauptursachen für regionale Unterschiede in der Arbeitslosigkeit angesehen wird. Wie das in einer Volkswirtschaft vorhandene Humankapital tatsächlich ausgenutzt wird, kann unter anderem an der Existenz von bestimmten Branchen liegen. Zeitliche Veränderungen und Diversität der Branchen haben in der Empirie meist signifikante Effekte für die Arbeitslosigkeit.

Auch Farhauer und Granato (2006) führen den Branchenmix als einen der wichtigsten Determinanten für regionale Disparitäten in der Beschäftigung in Deutschland an. Eine unmittelbare Beziehung mit dem Humankapital ist jedoch strittig. Blien, Südekum und Wolf (2006) untersuchen Beschäftigungseffekte in Westdeutschland und finden nur für das produzierende Gewerbe, nicht für Dienstleistungen, signifikante Effekte, die über einen starken Einfluss von Diversität der Wirtschaftsstruktur in einer Region hinaus wirksam werden. Dennoch hat der Dienstleistungssektor wachsende Beschäftigungszahlen vorzuweisen - fehlende direkte Wachstumseffekte können auch darin begründet sein, dass Dienstleistungen Wachstum in anderen Sektoren und Branchen induziert (Pugno, 2005).

Für Deutschland konstatieren empirische Arbeiten sowohl ein Stadt-Land-Gefälle wie ein Nord-Süd-Gefälle, was vornehmlich durch die Modernität der Wirtschaft und Verdichtung von Regionen erklärt wird, außerdem hat sich ein beträchtliches Ost-West-Gefälle manifestiert, das eine stärkere Ausprägung erreicht hat als das Nord-Süd-Gefälle (Bade, 2001). Dennoch ist eine weitergehende Divergenz des Humankapitals in ländlichen Gebieten nicht zu erwarten. Eckey, Kosfeld und Türck (2005) finden in Randregionen die höchste Grenzproduktivität der Beschäftigten mit Hochschulabschluss, was sie mit der (branchenspezifischen) Knappheit dieses Personals infolge seines Anstiegs in den Verdichtungsräumen erklären. Im Gegensatz zum Produktivitätswachstum ist das Beschäftigungswachstum jedoch weniger durch Spillover-Effekte nahe gelegener Regionen gekennzeichnet, die Prozesse sind vielmehr kleinräumig (Niebuhr, 2000).

Bezüglich des wirtschaftlichen Wachstums haben verschiedene Autoren für Europa, aber auch innerhalb Westdeutschlands, gezeigt, dass es zwischen innen eine absolute Konvergenz gibt. Jedoch haben einige Regionen, bei denen es sich vor allem um große Agglomerationsräume handelt, deutlich höhere Wachstumsraten (Geppert, Gornig \& Werwatz, 2005).

Im Hinblick auf die Verteilung des Humankapitals und seinen zeitlichen Verlauf lassen die Erkenntnisse aus der Regionalforschung keine eindeutigen Schlüssen zu. Die Wachstumstheorie und damit einhergehende Annahmen in der Regionalökonomie kommen 
jedoch zu dem Schluss, dass Humankapital für das Wachstum von Wirtschaft und Beschäftigung eine wichtige Voraussetzung ist.

\section{Die Bildungsvariablen}

Studien zur Bedeutung des Humankapitals verwenden eine Reihe verschiedener Bildungsvariablen. Die wichtigsten Variablen und die häufig geübte Kritik an diesen soll im nächsten Abschnitt zusammenfassend dargestellt werden. Batey (2002) weist wiederum darauf hin, dass verschiedene Indikatoren von Humankapital international zwischen den Ländern so variieren, dass keine Ländergruppe in allen Indikatoren führend ist. Je nachdem, welche Bildungsvariablen gewählt werden, ist also mit unterschiedlichen Ergebnissen im Bezug auf den Zusammenhang von Bildung und Beschäftigung zu rechnen.

Relative Bildungsausgaben. Diese Variable ist ein Input-Maß und wird in der Regel im Verhältnis zum Pro-Kopf-Einkommen betrachtet. Es steht für die Investitionsbereitschaft in Bildung in einem Land und für die quantitative Bedeutung der Bildung, für die mit einem quantitativen und qualitativen - Ertrag gerechnet wird. Kritisiert wird jedoch, dass der qualitative Komponente von Bildung nicht Rechnung getragen wird und eine Reihe anderer Störgrößen die Variable „verunreinigen" (als Beispiel können hier die Lehrergehälter genannt werden).

Einschulungsraten und durchschnittliche Bildungsjahre. Aus Einschulungsraten werden oftmals Angaben dazu berechnet, welcher Anteil der Bevölkerung einen Primär-, Sekundäroder Tertiärabschluss im Bildungssystem des Landes erworben hat. Auch als prozentuale Angabe bezüglich eines der drei Bildungsniveaus kann diese Variable verwendet werden. Dies ist unter anderem der Fall beim von Barro und Lee (1993) erstellten Datensatz, der beide Art von Angaben enthält. ${ }^{2}$ Der Datensatz dieser Autoren war in vielen empirischen Arbeiten die Quelle für die Bildungsangaben und wurde von den Autoren später in einer überarbeiteten Version veröffentlicht (Barro \& Lee, 2000). Sie konstruieren aus diesen Daten auch einen Humankapitalindex, der die erreichten Bildungsabschlüsse in durchschnittliche Jahre formaler Bildung umkodiert. Die Konstruktion eines solchen Humankapitalindex kann bei Existenz der entsprechenden Einzeldaten auch aus Kenntnissen der tatsächlich erreichten Bildungsabschlüsse gewonnen werden. Dies ist oft bei Befragungen der Fall, bei denen sich somit auf die Angaben der Befragten verlassen werden muss.

Anteil mit Hochschulabschluss. Der Anteil der Bevölkerung oder der Beschäftigten mit Hochschulabschluss findet hier noch einmal besonders Erwähnung, da diese Variable für die Innovationsfähigkeit von Ländern und Regionen herangezogen wird. Somit wird der theoretischen Größe "qualifizierte Arbeit" als Abgrenzung zum Arbeitsangebot Rechnung getragen.

Unterschiede zwischen männlichen und weiblichen Bildungsständen. Die Unterschiede zwischen den erreichten Bildungsabschlüssen von Frauen und Männern werden in internationalen Studien als Variable der Rückständigkeit betrachtet, die einen catch-upProzess zur Folge haben kann. Insofern ist interessant, ob ein solcher Effekt auch innerhalb eines Landes auftritt und ob eine Konvergenz zwischen den Regionen bezüglich der Differenzen der weiblichen und männlichen Werte beobachtbar ist.

Schüler-Lehrer-Ratio. Da oftmals davon ausgegangen wird, dass zu viele Schüler in einer Klasse sich negativ auf den Lernprozess auswirken, wird dieses Kriterium als

2 Einschulungsraten werden im "Human Development Index" gemeinsam mit der Alphabetisierungsquote auch als Variable herangezogen, die den Entwicklungsstand eines Landes kennzeichnet. 
Bildungsvariable herangezogen. Es ist aber keinesfalls empirisch gesichert, dass diese Annahme der Realität entspricht. Zu diesem Thema gibt es eine große Menge empirischer Literatur.

Eine Reihe weiterer Variablen findet sich in der empirischen Literatur und in letzter Zeit werden auch andere Wege gegangen. So eröffnen die Daten aus international vergleichenden Schulleistungstests wie PISA und TIMSS in den vergangenen Jahren neue Möglichkeiten (Coulombe, Tremblay \& Marchand, 2004). Andererseits finden auch Variablen wie Ergebnisse aus IQ-Tests Anwendung (Jones \& Schneider, 2006). Auf diese Weise wird eine stärker qualitativ orientierte Dimension berücksichtigt.

Iwahashi (2007) vermutet einen theoretischen Vorteil von Allgemeinbildung gegenüber der beruflichen Bildung, der abgeleitet wird aus der Annahme, dass eine längere allgemeine Ausbildung zu einer fundierteren Entscheidung zur Berufswahl führt, da mehr Kenntnisse über eigene Fähigkeiten erworben werden konnten, was zur Reduktion von Unsicherheiten führt. Iwahashi geht davon aus, dass entwickeltere Regionen mehr Möglichkeiten eröffnen und Informationen zu eigenen Fähigkeiten wertvoller werden. Empirisch weisen Bertocchi und Spagat (2003) einen positiven Effekt von allgemeiner Bildung im Verhältnis zu Berufsbildung nach, der jedoch erst ab einem bestimmten Entwicklungsniveau einsetzt. Amerikanische Untersuchungen sehen vor allem in der Highschool-Absolventenintensität nicht nur in langfristiger historischer Betrachtung eine besondere Bedeutung (Goldin \& Katz, 2001), sondern auch die stets schwierige Kausalität von Bildung und Beschäftigung hier recht eindeutig bei der Bildung angesiedelt (Bensi, Black \& Dow, 2004).

Die Untersuchung des Verhältnisses von Bildung zu Beschäftigung impliziert also die Verwendung einer Bildungsvariable und eine Wahl zwischen mehreren Variablen ist aufgrund fehlender Daten nicht immer möglich. Aus Kenntnis von Personendaten können jedoch mehrere Variablen und ihre Bedeutung für Beschäftigung und Wachstum gewonnen werden, die Aufschluss über den Stellenwert verschiedener Variablen ist. 


\section{Fragestellungen}

Die empirische Untersuchung, welche diesem Bericht zugrunde liegt, befasst sich mit einer Darstellung des regionalen Bildungsniveaus in Deutschland und des Zusammenhangs von Bildung und Beschäftigung auf regionaler Ebene. Die erste Frage lautet daher:

\section{(1) "Wie ist das Humankapital in Deutschland regional verteilt?"}

Im Einzelnen sollen des Weiteren in Anlehnung an obige Darlegungen folgende Hypothesen untersucht werden.

\section{(2) Das Humankapital in Deutschland ist nicht von Konvergenz gezeichnet.}

Wenn die Humankapitalvariable absolut konvergent ist, können mit ihr keine Disparitäten in den Wachstumsraten erklärt werden, wie sie im regionalen Zusammenhang für Deutschland für die Beschäftigungsentwicklung festgestellt wurden. Unter der Annahme der Bedeutung von Humankapital für das Wachstum von Wirtschaft und Beschäftigung wird daher nicht erwartet, dass die Konvergenzprozesse überwiegen.

\section{(3) Die Alterszusammensetzung hat keinen Einfluss auf den Humankapitalindex.}

Als Argument für die Investition in die Ausbildung wird unter anderem angeführt, dass infolge der Überalterung der deutschen Gesellschaft die Sorge besteht, dass nicht ausreichend viele gut ausgebildete junge Leute einem steigenden Humankapitalbedarf in Deutschland gegenüberstehen (Buttler \& Tessaring, 1993, Sinn, 2001). Zudem könnte aus der Abwanderung junger Leute ein Humankapitalmangel entstehen, zumal die junge Bevölkerung in Deutschland öfter höhere Bildungsabschlüsse erwerben. Aus der Theorie der Bildungsökonomie, ausgehend von Becker (1993), werden im Lebensverlauf abnehmende Grenzerträge für Humankapital zugrunde gelegt. Es wird jedoch angenommen, dass es in den Regionen Westdeutschlands keine gravierenden regionenspezifischen Unterschiede gibt, die dem (aggregierten) Trend in Westdeutschland entgegensteht.

\section{(4) Es ist in Deutschland ein Zusammenhang zwischen Bildung und} Beschäftigungswachstum auf regionaler Ebene nachzuweisen.

(4a) Die Branchenzusammensetzung spielt eine moderierende Rolle zwischen Bildung und Beschäftigung.

(4b) Neben den erreichten Bildungsabschlüssen sind insbesondere der Abiturientenanteil und der Anteil von Personen mit Hochschulabschluss Variablen, die mit Beschäftigung einhergehen.

Die Existenz eines Zusammenhangs von Bildung und Beschäftigungswachstum ist wenig umstritten. Dennoch konnte er nicht konsistent nachgewiesen werden und hängt auch mit der Wahl der Humankapitalvariable zusammen. In Anlehnung an die Argumente, die für allgemeine Bildung sprechen, soll überprüft werden, ob der Anteil von Abiturienten mit Beschäftigung zusammenhängt. Eine weitere Bildungsvariable, die insbesondere mit Innovationsfähigkeit in Verbindung gebracht wird, ist der Anteil von Personen mit Hochschulabschluss. Außerdem soll die Branchenbedeutung abgeschätzt werden, die eine wichtige Determinante für die regionale Beschäftigungsentwicklung darstellt und der somit eine moderierende Wirkung zugesprochen wird. 


\section{Die Daten}

\subsection{Datensätze}

Es werden vor allem die Daten der IAB - Regionalstichprobe, IABS-R01, verwendet. Die Regionalstichprobe als Scientific Use File ist ein Datensatz, welcher 2\% aller in Deutschland sozialversicherungspflichtig Beschäftigter umfasst. Der Datensatz ist insofern gut für Untersuchungen regionaler Dynamiken geeignet, da aufgrund seiner Größe eine zu geringe Zellenbesetzung bei der Betrachtung selbst tiefer Raumuntergliederungen nicht zu erwarten ist. So sieht die Stichprobe eine Raumaufteilung des Arbeitsortes auf Kreisebene vor. Ein weiterer Vorteil des Datensatzes ist die Tatsache, dass es sich um ein Panel handelt und dass er Daten von 1975 bis 2001 enthält. Insgesamt finden sich Informationen zu 1.293.819 Personen (1.112.761 in den alten und 181.058 in den neuen Bundesländern) und 21.041.596 Fällen in diesem Datensatz.

Da die Daten aus Angaben von Arbeitgebern stammen, zu deren Bericht diese verpflichtet sind, und die versicherungsrelevant sind, ist davon auszugehen, dass die Angaben zu den entsprechenden Angaben im Wesentlichen valide und vollständig sind. Darüber hinaus ist eine Vielzahl von Informationen zum Arbeitsmarkt enthalten, wie Informationen zur Branche und zum Beruf.

Weitere Regionaldaten, welche mit den aus den aggregierten Informationen aus dem Mikrozensus und der Regionalstichprobe verknüpft werden, entstammen dem INKARDatensatz (INKAR steht für "Indikatoren und Karten zur Raumentwicklung"). Es handelt sich dabei ebenfalls um amtliche Statistiken, welche vom Bundesamt für Bauwesen und Raumordnung (BBR) zur Verfügung gestellt werden. Dieses Bundesamt definiert auch die Raumordnungsregionen für das Bundesgebiet, welche folgend als Analyseräume genutzt werden.

\subsection{Die Bildungsvariable}

Die Bildungsvariable in der Regionalstichprobe ist in neun Kategorien unterteilt, die wie folgt kodiert wurden:

1 - ohne Berufsausbildung

2 - Volks-, Haupt-, Realschule mit Berufsausbildung

3 - Abitur ohne Berufsausbildung

4 - Abitur mit Berufsausbildung

5 - Fachhochschulabschluss

6 - Hochschulabschluss

7 - Ausbildung unbekannt

9 - Missing

Die den Personen zugeordneten Werte sind weniger vollständig und konsistent als beispielsweise die Angaben zum Lohn, da Bildungsauskünfte keine Relevanz für das Sozialsystem besitzen. So kommt es vor, dass der angegebene höchste erreichte Abschluss für eine Person zu einem späteren Zeitpunkt niedriger ist als ein zuvor angegebener, und dass die Anzahl der fehlenden Angaben vergleichsweise hoch ist.

Ein anderes Problem liegt darin, dass die Kategorie 2 „Volks-, Haupt-, Realschulabschluss mit Berufsausbildung" eine große Menge an Personen einschließt. Zur Umkodierung der Bildungsabschlüsse in Bildungsjahren ${ }^{3}$ ist dies prinzipiell unkritisch, weil alle

\footnotetext{
${ }^{3}$ Mehr hierzu später
} 
Schulabschlüsse jeweils 10 Jahre umfassen. Diese Kategorie ist sehr dominant, da sie etwa die Hälfte aller in der Stichprobe vertretenen Personen umfasst. Es ist daher zu erwarten, dass die durchschnittlichen Jahre formaler Schulbildung in den verschiedenen Regionen nahe dem dieser Kategorie zugeordneten Wert liegen und insofern recht homogen sind. Eine weitere Abstufung innerhalb dieser großen Gruppe wäre jedoch künstlich. So wäre denkbar, in Kombination mit der Variable "Stellung im Beruf" zusätzliche Varianz zu erzeugen. Diese Variable unterscheidet vor allem zwischen Facharbeitern, NichtFacharbeitern und Angestellten. Jedoch befindet sich auch die Merkmalsausprägung „Teilzeitbeschäftigung" in dieser Variable, ohne diese Personen einer eindeutigeren Stellung zuzuordnen. Der Anteil ist relativ hoch: im Jahr 2001 sind 22,8\% der Personen in dieser Kategorie in Teilzeit tätig (1975 sind es nur 6,1\%). Darüber hinaus kann keine Aussage darüber gemacht werden, ob Personen ausbildungsadäquat beschäftigt sind oder welche Ausbildungsintensität für die einzelnen Stellungen typisch sind.

Einige Ungleichgewichte sind für die Bildungsvariable besonders bei den nicht-deutschen Beschäftigten zu beobachten. Ausländer haben überproportional häufig keine Berufsausbildung oder einen fehlenden Wert bei der Bildungsvariablen. ${ }^{4}$ Während in der Gesamtdatei $7,9 \%$ der Fälle Ausländer sind, fehlen für diese Gruppe bei den Bildungsangaben 17,5\% der Gesamtstichprobe. Für 14,9\% der fehlenden Werte existieren keine Angaben zur Nationalität, $10,7 \%$ sind es in der Gesamtstichprobe. Ausländer mit Volks-, Haupt-, Realschulabschluss und Berufsausbildung sind im Vergleich mit deutschen Staatsbürgern zwar prozentual gesehen häufiger Nichtfacharbeiter, jedoch stellen sie nur etwa ein Neuntel aller Nichtfacharbeiter dar. Auffällig ist auch, dass sie deutlich seltener Angestellte sind. Auch unabhängig von der Nationalität sind Missings überproportional den Nichtfacharbeitern zuzuordnen sowie den Teilzeitbeschäftigten ohne Arbeitslosenversicherung.

Fitzenberger, Osikominu und Völter (2005) drücken ihre Beobachtungen zu fehlenden Werten der Bildungsvariable in Wahrscheinlichkeiten aus, argumentieren aber, dass diese zwar in den meisten Fällen signifikant, jedoch angesichts 7,8\% fehlender Werte in einer von innen gewichteten Stichprobe nicht besonders groß sind. Die Autoren haben zur Verbesserung der Bildungsvariable vier verschiedene Imputationen durchgeführt, welche in die IAB-Stichprobe eingearbeitet wurde. Die so verbesserten Daten stehen seit November 2006 zur Verfügung.

Diese Imputationen beruhen auf unterschiedlichen Annahmen zu den Gründen fehlender Werte. Sie reduzieren fehlende Werte dadurch auf 1,9\% bis 3,2\% der Gesamtstichprobe je nach Imputation bzw. auf $1,2 \%$ bis $2,1 \%$ der gewichteten Stichprobe. Darüber hinaus ersetzen sie unplausible und inkonsistente Werte, wodurch sich die Struktur der Variablen ändert.

Es wurden alle vier Imputationen von Fitzenberger, Osikominu und Völter (2005) in Jahre formaler Bildung umkodiert (siehe den nächsten Abschnitt). So konnte anhand der Interkorrelation der verschiedenen Variablen überprüft werden, wie stark sie miteinander verbunden sind. Die Korrelationen über alle Jahre und Raumordnungsregionen betragen zwischen .993 (Imputation $2 \mathrm{a}$ und 4) und .998 (2a und 2b). Letzteres ist nicht verwunderlich, da $2 \mathrm{a}$ und $2 \mathrm{~b}$ mit denselben Basisannahmen vorgenommen wurden. Auch die Anzahl der Fälle pro Raumordnungsregion und Jahr sind in fast allen Fällen quasi perfekt korreliert. Nach Fitzenberger, Osikominu und Völter sind die Unterschiede zwischen den Imputationen so gering, dass bei einer Wahl zwischen den Variablen im Prinzip unbedeutend ist, zugunsten welcher Variable die Entscheidung ausfällt. In der vorliegenden Untersuchung wurde daher die dritte Imputation gewählt, da diese auf Annahmen beruht, die weniger stark sind als die Annahmen der Imputationen 1 und 2, aber bezüglich der

\footnotetext{
${ }^{4} \mathrm{Zu}$ fehlenden Werten wurden im Weiteren die Kategorien "Missing" und „Ausbildung unbekannt" zusammengefasst.
} 
Fallzahl stärker mit diesen zusammenhängt als die vierte Imputation. Der mittlere Unterschied zu den anderen Imputationen beträgt .079 Jahre im Vergleich zur ersten, $.046 / .049$ zu den zweiten und .028 zur vierten Variablen.

Bei der dritten Imputation wird angenommen, dass die Häufigkeit der gleichen Angabe zum Bildungsstand von Reliabilität zeugt und diese größer ist, wenn verschiedene Arbeitgeber dieselbe Angabe gemacht haben als wenn ein Arbeitgeber häufig die gleiche Angabe gemacht hat. Zudem wird eine Korrektur der Angaben seitens der Arbeitgeber als zusätzliches Reliabilitätskriterium herangezogen.

\subsection{Umkodierungen der Bildungsvariable}

Zum besseren Vergleich der Regionen wird jeder Region ein einziger Zahlenwert zugeordnet, der alle Abschlüsse der Variable „BILD” (Bildung) für die Gesamtzahl der Beschäftigten in einer Region abbildet. Die Tatsache, dass die Bildungsvariablen in der Regionalstichprobe auf eine einzige Variable beschränkt sind, grenzt die Analysemöglichkeiten ein. Dennoch werden im Weiteren verschiedene Varianten als Humankapitalindikatoren angewendet, welche verschiedene Wirkungszusammenhänge zwischen Bildung und Beschäftigung aufzeigen. Dabei soll verglichen werden, welche der verschiedenen Variablen in besonderem Zusammenhang zur Beschäftigung stehen. Zunächst wird der Zusammenhang und die Verteilung der formalen Bildung mit dem in der Bildungsökonomie besonders häufig verwendeten Indikator "durchschnittliche Bildungsjahre" überprüft. Hierfür werden Umkodierungen der Abschlussniveaus in Jahre notwendig.

Die Kodierung der IAB-Daten wurde bis auf die Kategorien "1" und "2" aus der Publikation von (Ostermeier \& Blossfeld, 1998) übernommen. Daraufhin wurden diese Umkodierungen der Variable „BILD" vorgenommen:

Tabelle 1: Kodierungen der Humankapitalvariable in Jahre formaler Bildung

\begin{tabular}{|l|c|c|}
\hline $\begin{array}{c}\text { Merkmalsausprägung } \\
\text { IABS-R01 }\end{array}$ & $\begin{array}{c}\text { Ostermeier \& } \\
\text { Blossfeld (1998) }\end{array}$ & $\begin{array}{c}\text { Eigene } \\
\text { Umkodierungen }\end{array}$ \\
\hline ohne Berufsausbildung & $10 / 9 / 8^{1}$ & 9 \\
\hline $\begin{array}{l}\text { Volks-, Haupt-, } \\
\text { Realschule mit } \\
\text { Berufsausbildung }\end{array}$ & $12 / 11^{2}$ & 12 \\
\hline $\begin{array}{l}\text { Abitur ohne } \\
\text { Berufsausbildung }\end{array}$ & 13 & 13 \\
\hline $\begin{array}{l}\text { Abitur mit } \\
\text { Berufsausbildung }\end{array}$ & 15 & 15 \\
\hline Fachhochschulabschluss & 17 & 17 \\
\hline Hochschulabschluss & 19 & 19 \\
\hline
\end{tabular}

18 Jahre: ohne Schulabschluss und ohne Berufsausbildung; 9 Jahre: Hauptschulabschluss ohne Berufsausbildung; 10 Jahre: Mittlere Reife ohne Berufsausbildung

211 Jahre: Hauptschulabschluss mit Berufsausbildung; 12 Jahre: Mittlere Reife ohne Berufsausbildung

Da für die beiden ersten Ausprägungen keine Vergleichswerte aus den beiden zitierten Studien vorliegen, wurden diese Werte selbst gewählt. In der Kategorie „1" sind sowohl die Personen enthalten, die einen Volks-, Real- oder Hauptschulabschluss erworben haben, aber auch die Personen, die überhaupt keinen Abschluss vorweisen können. Aus diesem Grund wurde diese Kategorie mit 9 Jahren umkodiert. Es wurde für die Ausprägung "2" 
eine Umkodierung gewählt, bei der der Unterschied zwischen den „2" (Volks-, Haupt-, Realschulabschluss mit Berufsausbildung) und ",3" (Abitur ohne Berufsausbildung) nur ein Jahr beträgt. Die Unterschiede im Vergleich mit anderen Kodierungen sind aber als eher gering anzusehen. Ein Großteil der Regionen - etwa zwei Drittel - verändert seinen Rang innerhalb der 97 Raumordnungsregionen trotz einer relativen geringen Varianz zwischen den Werten zu den einzelnen Regionen nicht.

Auf eine Gewichtung nach Länge der Spells wurde zugunsten einer anderen Variante verzichtet. So wurde von jeder Person nur ein einziger Spell pro Jahr zugelassen und der Einfachheit halber wurde stets die erste Meldung verwendet. Dies ist vor dem Hintergrund der gegebenen Fragestellung auch dadurch zu rechtfertigen, dass eine Person lediglich einfach pro Jahr im aggregierten Wert zur Bildung in einer Region erscheinen sollte. ${ }^{5}$

\subsection{Betrachtete Personen}

Es wurden in allen Analysen Personen berücksichtigt, die volljährig sind und unterhalb des gesetzlichen Renteneintrittsalters liegen. In der IABS-Regionalstichprobe besteht aber das Problem, dass es über 62Jährige gibt, für die nicht eine Kodierung sondern das tatsächliche Alter vorliegt, in anderen Fällen eine Kodierung gewählt wurde. Daher wurden nur die 6265Jährigen eingeschlossen, für die eine Angabe zum Geburtsjahr vorliegt. Geringfügig Beschäftigte, für die Daten ab 1999 vorliegen, wurden ausgeschlossen.

\subsection{Die regionale Variable}

Die Analyse wurde auf der Ebene der Raumordnungsregionen vorgenommen. Es gibt 97 Raumordnungsregionen in Deutschland. Diese Aufgliederung erlaubt es, mit den üblichen statistischen Verfahren zu arbeiten und durch Desaggregation einen genaueren Blick auf die sehr unterschiedlich strukturierten Regionen innerhalb eines Bundeslandes zu werfen. Im IAB-Datensatz sind die Regionen sogar noch detaillierter erfasst, werden aber zum Zweck der hier veröffentlichten Analyse zu Raumordnungsregionen zusammengefasst.

Die Raumordnungsregionen sind durch synthetische Regionalisierung entstanden. Sie „stellen politisch motivierte Planungsregionen der Länder dar, die auf der räumlichen Grundeinheit ,Kreis' aufbauen und die Abgrenzungen der Regierungsbezirke als Untereinheiten der Länder nicht verletzen." (Hoffmeyer-Zlotnik, 2000). In der IABRegionalstichprobe werden die Kreise angegeben, in denen die entsprechende Person beschäftigt ist. Diese wurden den Raumordnungsregionen zugeordnet.

Eine Besonderheit betrifft die Region „Berlin". Es liegen hier Daten vor der Wiedervereinigung aus dem westdeutschen Teil Berlins vor, die später mit dem anderen Teil Berlins erweitert wurden. 74 weitere Regionen sind Westdeutschland zuzuordnen. 22 weitere Regionen gehören zu Ostdeutschland, weshalb die Daten nur über eine viel kürze Zeit vorliegen.

\footnotetext{
${ }^{5}$ Um zu überprüfen, wie sich durch diese Veränderung der Datensatz verändert, habe ich die aggregierten Humankapitalwerte der nicht-bereinigten mit der bereinigten Version verglichen. Hierfür wurden für beide Fälle die Dezentile und Quintile berechnet. Bei den Dezentilen bleiben $78,4 \%$ im gleichen Dezentil, 20,6\% verschieben sich um 1 Dezentil und 1\% um 2 Dezentile. Bei den Quintilen gibt es noch weniger Verschiebungen: $87,6 \%$ bleiben in der gleichen Kategorie und die übrigen $12,4 \%$ verschieben sich jeweils um eine Kategorie. Die durch die Bereinigung erzielten Veränderungen sind also eher gering.
} 


\section{Ergebnisse}

\subsection{Regionale Verteilung des Humankapitals in Deutschland}

Die regionale Verteilung der Variable Humankapital wird als Mittelwert der Jahre formaler Bildung derjenigen sozialversicherungspflichtig Beschäftigten angegeben, für die Humankapitalangaben vorliegen. Es soll begonnen mit der Darstellung der Werte aus dem Jahr 2001 werden, dem jüngsten in der Datenbasis vorhandenen Jahr. Die Mittelwerte liegen zwischen 11,84 Jahren (Region Landshut) und 13,35 Jahren (Region München). Darin gingen mindestens Werte von 1338 Personen (Region Schleswig-Holstein Süd-West) und höchstens von 22894 (Region Rhein-Main) ein. Somit liegen die Regionen im äußersten Fall über $12,8 \%$ in ihrer (wie oben definierten) Humankapitalausstattung auseinander.

Auffällig ist dabei, dass zu den Regionen mit der geringsten Humankapitalausstattung viele der südlichen Region zählen. Es handelt sich dabei in vielen Fällen um ländliche Regionen. Es werden zwei Merkmale als Indikatoren für die Unterscheidung zwischen ländlichen und städtischen Regionen herangezogen: der Anteil von für Landwirtschaft genutzter Fläche und die Bevölkerungsdichte. Regionen, die in beiden Indikatoren in der unteren Hälfte liegen, wurden als „ländlich" bezeichnet. Die Ländlichkeit der Regionen ist jedoch nicht allein ausschlaggebend, zumal einige der ländlichsten Regionen wie Schleswig-Holstein Nord sich im Bereich des Mittelwertes befinden. Abbildung 1 stellt die Einwohnerdichte und den Humankapitalindex für das Jahr 2001 dar.

Der Mittelwert über alle 97 Regionen beträgt 12,36 Jahre (berechnet auf Grundlage der bereits aggregierten Daten). Wenn man nur die 74 westdeutschen Regionen (ohne Berlin) betrachtet, ist der Bestand im Schnitt 12,29 Jahre - liegt also darunter.

Im Jahr 1975, in dem die Datenbasis beginnt, liegen die durchschnittlichen Jahre formaler Bildung deutlich niedriger: 10,57/10,58/10,59 Jahre sind die niedrigsten Werte für die Regionen Donau-Wald, Schwarzwald-Baar-Heuberg und Neckar-Alb. Auch die Region Landshut, die in 2001 den niedrigsten Wert aufweist, liegt nahe diesen Werten (10,62 Jahre). Mit 11,48 Jahren ist Hamburg die Region mit dem größten Wert, gefolgt von der Region München mit 11,41 Jahren. Somit liegt die prozentuale Differenz zwischen der Region mit der geringsten und der Region mit der höchsten Ausstattung bei 8,6\%. Dieser prozentuale Unterschied hat sich also im Zeitverlauf deutlich erhöht. 


\section{Abbildung 1: Humankapitalindex und Einwohnerdichte in Deutschland}

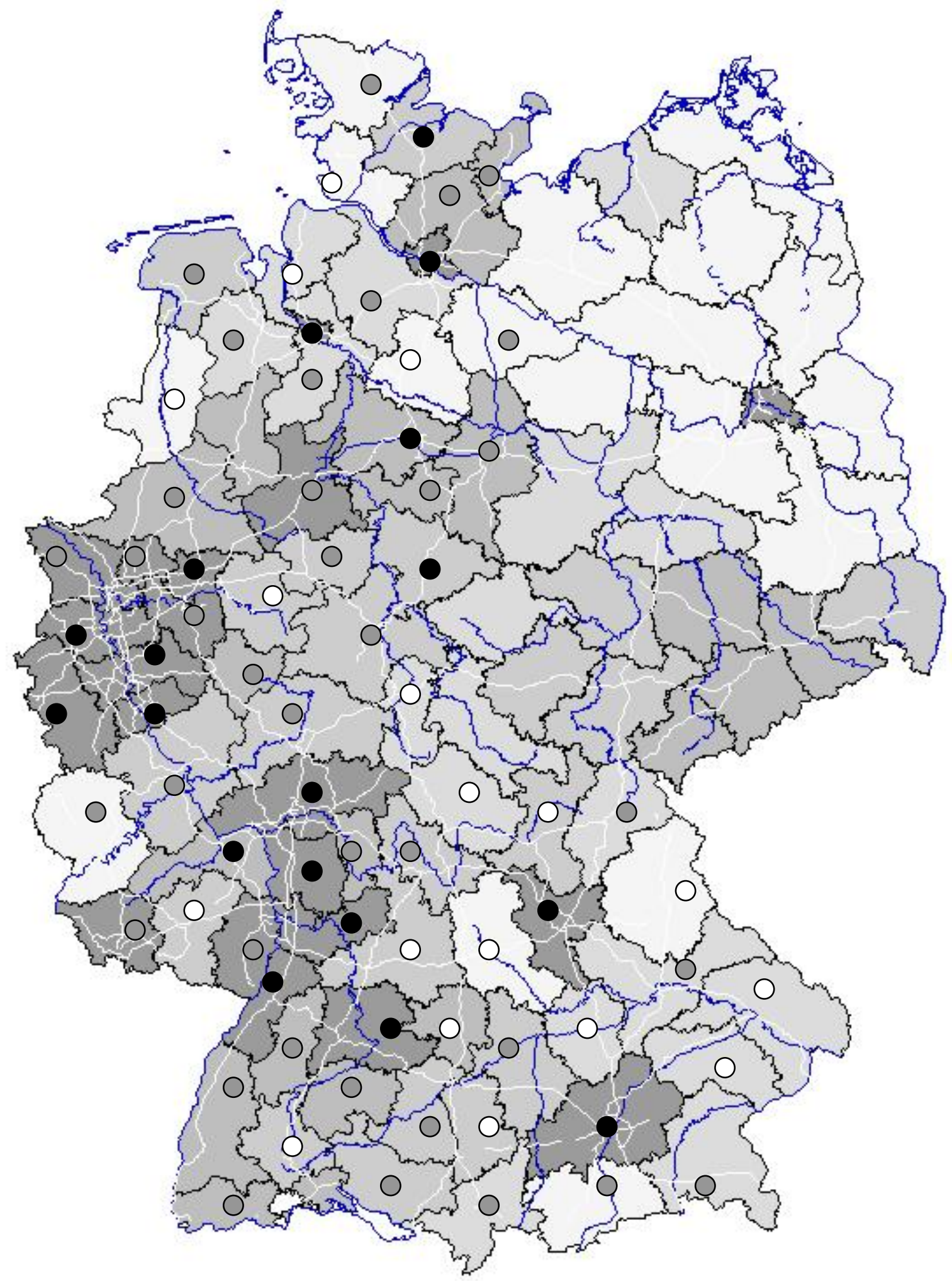

- Humankapitalindex im oberen Viertel

$\bigcirc$ Humankapitalindex im unteren Viertel

Einwohner je $\mathbf{k m}^{\mathbf{2}}$

Humankapitalindex in der Mitte

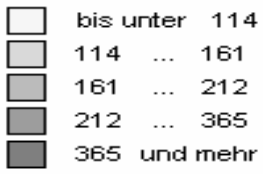




\subsubsection{Zeitlicher Verlauf der Humankapitalausstattung}

Zur Beantwortung der zweiten Hypothese wird die zeitliche Entwicklung des Humankapitalindexes näher in Augenschein genommen. Zur Betrachtung der Veränderung der Humankapitalausstattung seit 1975 wurden nur die Daten aus westdeutschen Regionen übernommen, für welche alle seit 1975 Daten vorliegen. Die Veränderung in Jahren des Humankapitals beträgt im geringsten Fall 1,01 Jahre und im höchsten Fall 1,94 Jahre, das bedeutet prozentuale Veränderungen von mindestens 9,13\% (Region Bremerhaven) und höchstens 12,78\% (Region München). 50\% liegen zwischen 1,20 Jahren und 1,44 Jahren. Die Region "Emsland" (1,01 Jahre) hat die geringste Verbesserung aufzuweisen und München (1,94 Jahre) die größten Verbesserungen.

Die folgende Abbildung zeigt, wie die zwei Regionen mit den zwei höchsten und den zwei niedrigsten Werten in 2001 und 1975 sich entwickelt haben.

\section{Abbildung 2: zeitlicher Verlauf des Humankapitalindexes 1975-2001 in ausgewählten Raumordnungsregionen}

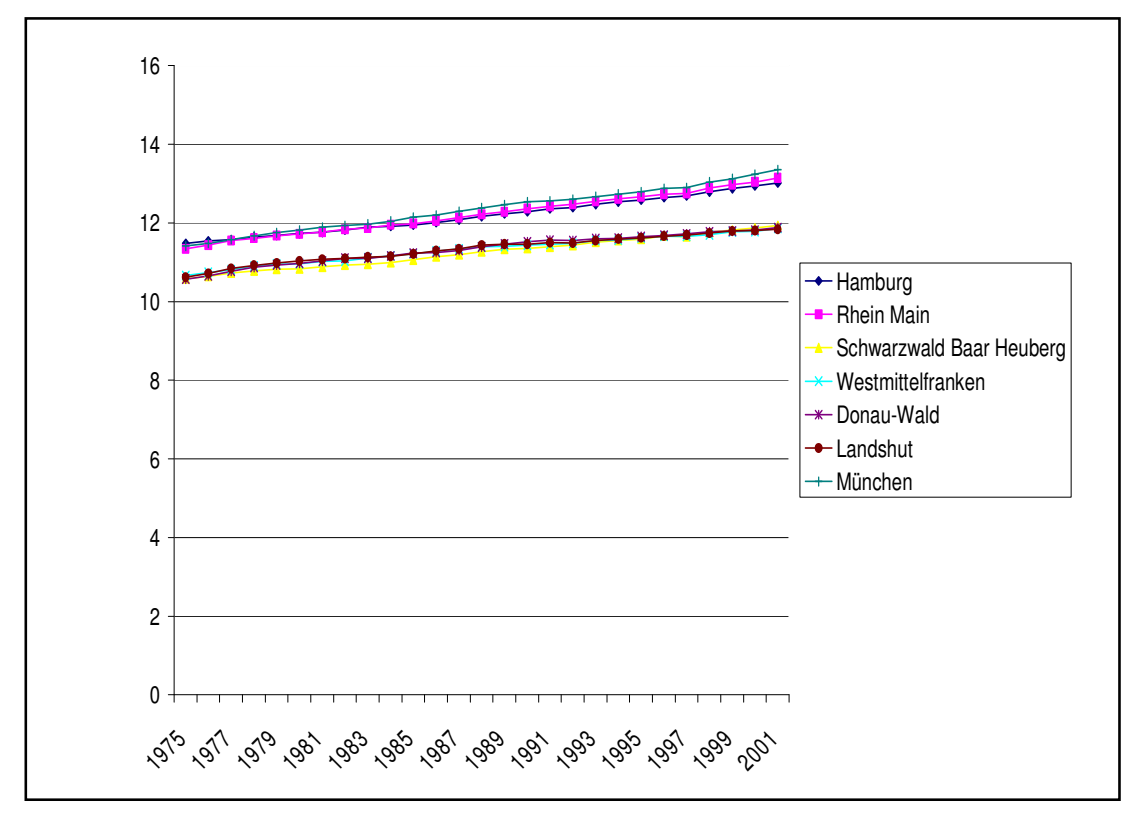

Diese Abbildung sowie die erwähnte größere Diskrepanz zwischen den Regionen mit den niedrigsten und höchsten Humankapitalindizes 1975 und $2001 \mathrm{im}$ Vergleich weisen darauf hin, dass keine absolute Konvergenz des Humankapitalindexes zwischen den Regionen erkennbar ist. Die Abbildungen 3a) b) und c) bilden das Verhältnis ab, welches der Schuljahresbestand in den Jahren 1975, 1985 und 1995 zur Veränderung des Bestandes bis zum Jahre 2001 in Jahren hat: Es ist deutlich zu erkennen, dass Regionen mit einem hohen Bestand in 1975 auch tendenziell eine höhere Verbesserung aufweisen können. Die entsprechende Korrelation bestätigt eine zum Teil hohe Signifikanz dieses Zusammenhangs für verschiedene Jahre: $r=.220$ (nicht signifikant) für $1975, r=.581$ für 1985 und $r=$ .659 für 1995 (jeweils hochsignifikant). 


\section{Abbildungen 3 a) zeitliche Veränderung des Humankapitalindexes 1975-2001, b) $1985-2001$ und c) $1995-2001$}

3a)
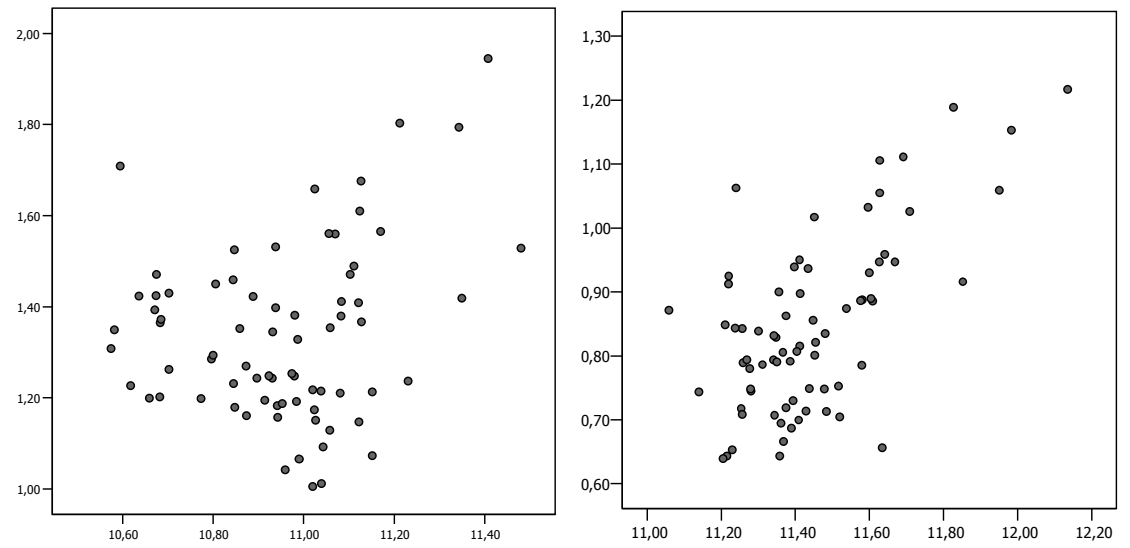

3c)

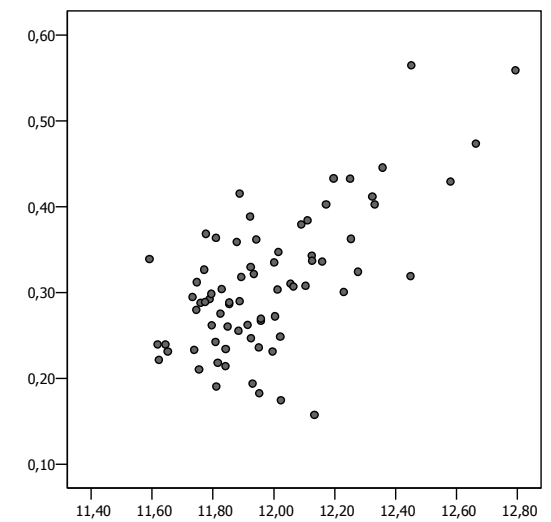

$3 b)$

Diese Veränderung lässt darauf schließen, dass die Schere zwischen den Regionen mit starker und geringer Humankapitalintensität größer wird. Ein formaleres Kriterium kann hierüber mehr Aufschluss geben. Dazu kann folgende Regressionsgleichung herangezogen werden:

$$
\ln \left(\mathrm{HK}_{2001} / \mathrm{HK}_{1975}\right)=\alpha+\beta \ln \left(\mathrm{HK}_{1975}\right)^{6},
$$

wobei die Veränderung der Humankapitalindices (HK) in den Jahren 1975 und 2001 auf das Ausgangsniveau regressiert wird. Die Veränderung des Humankapitalindexes wird als durchschnittliche jährliche Veränderung berechnet, die somit ein stetiges Wachstum darstellt. Die Ergebnisse sind gemischt, je nachdem, von welchem Jahr an die Konvergenz berechnet wird.

$$
\begin{array}{ll}
\ln \left(\mathrm{HK}_{2001} / \mathrm{HK}_{1975}\right)=-6,282+0,229 \ln \left(\mathrm{HK}_{1975}\right) & \left(\mathrm{R}^{2}=.084\right) \\
\ln \left(\mathrm{HK}_{2001} / \mathrm{HK}_{1985}\right)=-16,478+0,661 \ln \left(\mathrm{HK}_{1985}\right) & \left(\mathrm{R}^{2}=.437\right) \\
\ln \left(\mathrm{HK}_{2001} / \mathrm{HK}_{1995}\right)=-2,451-0,086 \ln \left(\mathrm{HK}_{1995}\right) & \left(\mathrm{R}^{2}=.007\right)
\end{array}
$$

Dass keine Konvergenz vorliegt, deutet die fehlende Signifikanz des $\beta$-Koeffizienten an. Dies trifft jedoch nicht auf den Zeitraum vom 1985 bis 2001 zu, jedoch ist das Vorzeichen entgegen der Erwartung im Falle von Konvergenz positiv, was auf eine Divergenz schließen lässt. Die sehr kleine erklärte Varianz in den anderen Zeiträumen lässt ebenfalls die Schlussfolgerung zu, dass die Regionen nicht konvergieren.

6 Der Logarithmus wird verwendet, weil für die Wachstumsraten typischerweise eine Exponentialfunktion angenommen wird, die so linearisiert wird (siehe auch Abschnitt 6.7). 


\subsubsection{Regionale Verteilung des Humankapitals für Männer und Frauen}

Es wurde für Männer und Frauen jeweils pro Jahr und Region ein Humankapitalindex errechnet und mit den Daten für beide Geschlechter verglichen. Im zweiten Schritt wurden die Veränderungen zwischen den Jahren 1975 bis 2001 für alle drei Fälle berechnet: Männer, Frauen, beide Geschlechter. Damit wurden zunächst drei Graphen erstellt (siehe Abbildungen $4 a$ ) und $b$ ), nur Westdeutschland).

Graph 4a) zeigt, dass für 1975 die Regionen mit hohem Wert für Männer auch für Frauen einen höheren Wert haben und umgekehrt, mit einigen Ausreißern in der Mitte. Für das Jahr 2001 sieht der Graph ähnlich aus wie für 1975, jedoch mit einer etwas geringeren Streuung (mit hohen Werten für beide, Abbildung 4b). Eine Konvergenz ist also bereits auf dieser einfachen Beschreibungsebene zu beobachten.

\section{Abbildungen 4 a) Verhältnis des Humankapitalindexes von Männern und Frauen 1975 und b) 2001}
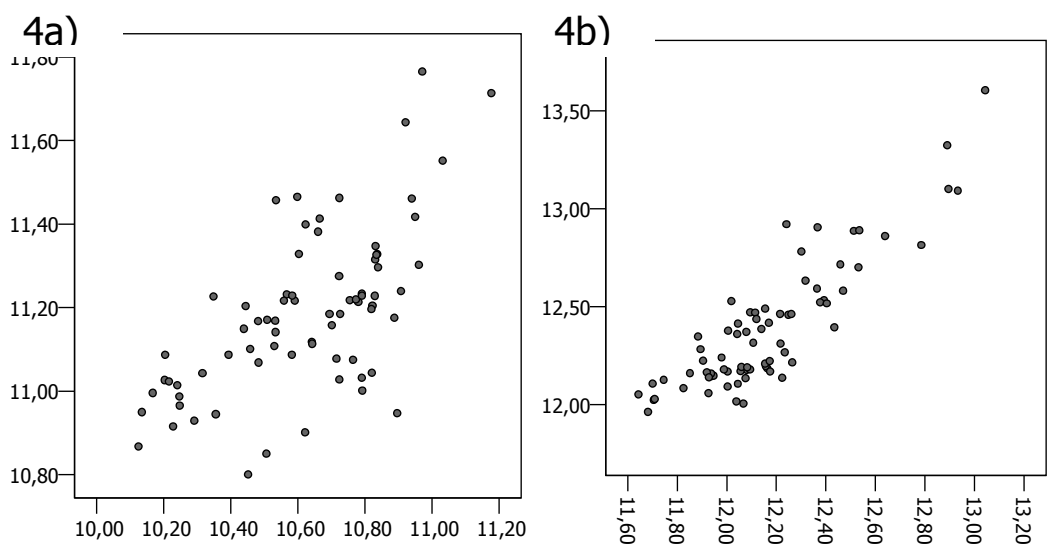

Donau-Wald und Schwarzwald-Baar-Heuberg sind die beiden Regionen, welche 1975 die niedrigsten Werte für die weibliche Bildungsvariable besitzen. Der Humankapitalindex liegt hier bei 10,12 bzw. 10,13 Jahren. Die höchsten Werte liegen knapp über 11 Jahren, die hier Hamburg mit 11,18 Jahren und Bremen mit 11,03 Jahren aufweisen. 26 Jahre später befinden sich die geringsten Indices für die weiblichen Beschäftigten in den nahe gelegenen Gebieten Landshut und Westmittelfranken. Es sind die Regionen München und Bonn, wo die weibliche Bildungsvariable den höchsten Wert annimmt (München: 13,04 Jahre; Bonn: 12,93 Jahre). Tabelle 2 listet die fünf Regionen mit den jeweils höchsten und niedrigsten Werten für die Humankapitalindices auf.

Die Differenzen zwischen weiblichen und männlichen Indices und ihre regionale Verteilung sind insofern von Interesse, da die Regionen mit deutlichem Vorsprung der männlichen Indices in der Literatur zu internationalen Studien als verhältnismäßig rückständig bezeichnet werden. Die Differenzen sind 1975 alle positiv (Männer haben höhere Indices als Frauen) und betragen zwischen 0,05 Jahren (Region Emscher-Lippe) und 0,92 Jahren (Industrieregion Mittelfranken). Die niedrigen Werten beim weiblichen Index und die Differenzvariable sind hochsignifikant mit $r=.62$ korreliert (38\% erklärte Varianz). 
Tabelle 2: Die 5 Regionen mit den höchsten und niedrigsten Werten in den geschlechterbezogenen Humankapitalindices

\begin{tabular}{|l|l|l|l|l|}
\hline Pos. & $\begin{array}{l}\text { Weiblicher Index } \\
\mathbf{1 9 7 5}\end{array}$ & $\begin{array}{l}\text { Weiblicher Index } \\
\mathbf{2 0 0 1}\end{array}$ & $\begin{array}{l}\text { Differenz } \\
\text { weiblicher- } \\
\text { männlicher Index } \\
\mathbf{1 9 7 5}\end{array}$ & $\begin{array}{l}\text { Differenz } \\
\text { weiblicher- } \\
\text { männlicher Index } \\
\mathbf{2 0 0 1}\end{array}$ \\
\hline 1 & Hamburg & München & Emscher Lippe & Emscher Lippe \\
\hline 2 & Bremen & Bonn & Trier & Südheide \\
\hline 3 & München & Hamburg & $\begin{array}{l}\text { Schleswig-Holstein } \\
\text { Süd-West }\end{array}$ & $\begin{array}{l}\text { Schleswig-Holstein } \\
\text { Nord }\end{array}$ \\
\hline 4 & $\begin{array}{l}\text { Schleswig-Holstein } \\
\text { Ost }\end{array}$ & Rhein-Main & Siegen & Münster \\
\hline 5 & Bonn & Köln & Arnsberg & Emsland \\
\hline$\ldots$ & \multicolumn{5}{|l|}{} \\
\hline 70 & Oberfranken Ost & Donau-Wald & Neckar-Alb & Mittlerer Oberrhein \\
\hline 71 & $\begin{array}{l}\text { Bayrischer } \\
\text { Untermain }\end{array}$ & Oberpfalz Nord & Mittlerer Oberrhein & $\begin{array}{l}\text { Bodensee } \\
\text { Oberschwaben }\end{array}$ \\
\hline 72 & Neckar Alb & $\begin{array}{l}\text { Schwarzwald-Baar- } \\
\text { Heuberg }\end{array}$ & Oberfranken Ost & Stuttgart \\
\hline 73 & $\begin{array}{l}\text { Schwarzwald-Baar- } \\
\text { Heuberg }\end{array}$ & Landshut & $\begin{array}{l}\text { Bodensee } \\
\text { Oberschwaben }\end{array}$ & München \\
\hline 74 & Donau-Wald & Westmittelfranken & $\begin{array}{l}\text { Industrieregion } \\
\text { Mittelfranken }\end{array}$ & $\begin{array}{l}\text { Industrieregion } \\
\text { Mittelfranken }\end{array}$ \\
\hline
\end{tabular}

Im Jahr 2001 ist ein großer Teil der Geschlechterunterschiede verschwunden, für fünf Regionen ist das Vorzeichnen sogar negativ (Frauen mit einem leicht höheren Index). Die Differenzen reichen von -0,09 (Emscher-Lippe) bis zu 0,68 (Industrieregion Mittelfranken). Die Differenzwerte der beiden Jahre korrelieren hochsignifikant miteinander $(r=.75)$, jedoch gibt es keine signifikante Korrelation zwischen den Differenzwerten und den weiblichen Indices im Jahr 2001. In den Regionen, in denen 2001 der weibliche Index sehr gering war, war demnach auch der männliche Index nicht weiter vom weiblichen entfernt als in anderen Regionen.

Diese Ergebnisse entspricht damit den Befunden zu internationalen Daten von Weber (1998) insofern, als dass auch hier eine absolute Konvergenz beobachtet werden kann. Dennoch ist auch sichtbar, dass die Konvergenz innerhalb der Regionen, aber nicht zwischen den Regionen statt findet. Die Steigerungen gelten in der Regel für beide Geschlechter, wenn auch in unterschiedlicher Ausprägung. Wendet man das Konvergenzkriterium (siehe 6.2.1) auch hier an, stellt sich dieses so dar:

$$
\ln \left(\partial_{2001} / \partial_{1975}\right)=\alpha+\beta \ln \left(\partial_{1975}\right),
$$

wobei die Veränderung der Differenzen zwischen männlichen und weiblichen Indices ( $\circlearrowright$ ) in den Jahren 1975 und 2001 auf das Ausgangsniveau regressiert wird.

$$
\ln \left(\partial_{2001} / \partial_{1975}\right)=-1,059-0,144 \ln \left(\partial_{1975}\right) \quad\left(R^{2}=.021\right)
$$

Wie bei gemeinsamer Betrachtung beider Geschlechter bezeugt das Ergebnis, dass zwischen den Regionen keine Konvergenz vorliegt. Damit unterstützt dieses Ergebnis die Vermutung, dass eine Konvergenz innerhalb der Regionen sich nicht in einer Konvergenz zwischen Regionen niederschlägt. 
Eine Sonderstellung bilden in beiden Geschlechtergruppen die ostdeutschen Regionen. Im nächsten Abschnitt wird daher näher auf den Ost-West-Vergleich eingegangen.

\subsection{Regionale Verteilung im Vergleich zwischen Ost und Westdeutschland}

Dass die Regionen in den neuen deutschen Bundesländern nicht ohne weiteres mit den westdeutschen Regionen vergleichbar sind, liegt auf der Hand. Das Bildungssystem in der DDR war anders strukturiert und verfolgte andere Ziele. Eine Vielzahl der in Ostdeutschland Beschäftigten wurde in diesem Bildungssystem ausgebildet, und auch heute ist das Abitur nach 12 Jahren erreicht. Dieser Abschnitt hat zum Ziel, auf der deskriptiven Ebene kurz zu beschreiben, wie sich die östlichen von den westlichen Regionen unterscheiden. Zusammenhangsmaße in der weiteren Analyse werden aufgrund dieser Unterschiede nur für die 74 westdeutschen Regionen berechnet (ohne Berlin). Weitere Untersuchungen zu den Befunden zu den ostdeutschen Regionen sind späteren Analysen vorbehalten.

In Ostdeutschland offenbaren sich die systembedingten Unterschiede in den Humankapitalvariablen: Der Humankapitalindex ist in Ostdeutschland höher, sowohl bezüglich der weiblichen als auch der männlichen Bildungsvariable. Der Mittelwert der 74 deutschen Raumordnungsregionen beträgt im Jahr $200112,49^{7}$ in Westdeutschland und 12,47 in Ostdeutschland.

In West- wie Ostdeutschland nimmt der Humankapitalindex zu, wenngleich er im Osten langsamer wächst als im Westen (siehe Abbildung 5). Dieser Unterschied kann Folgendes zur Ursache haben:

(1) ein Teil gut ausgebildeter Angestellter ist in den Westen gezogen ist,

(2) weniger junge Menschen mit besserer Ausbildung drängen auf den Arbeitsmarkt und/oder

(3) es handelt sich um einen gewöhnlichen Konvergenzprozess.

\section{Abbildung 5: Humankapitalindex von Ost- und Westdeutschland im Vergleich}
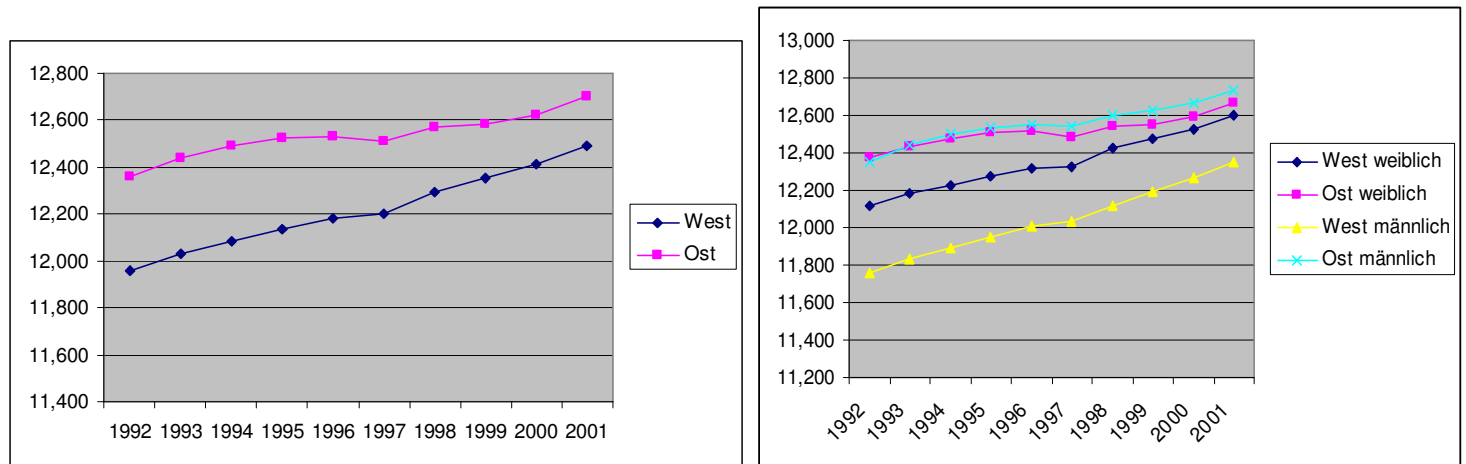

Betrachtet man nur die unter 35Jährigen, so ist festzustellen, dass das Bildungsniveau in Ostdeutschland sich beinahe spiegelverkehrt zum Humankapitalindex in Westdeutschland verhält (Abbildung 6). Während das Niveau kurz nach der Wende im Osten höher liegt als im Westen, so ist es ab 1996 niedriger. Dies könnte zwei der erwähnten Vermutungen stützen: Es werden weniger Menschen gut ausgebildet und die gut Ausgebildeten wandern mangels Arbeitsplätzen in den Westen aus. Der Konvergenzannahme widerspricht das Ergebnis, da sich die beiden Regionen gegenläufig entwickelt haben.

\footnotetext{
${ }^{7}$ Da dieser Wert auf Basis der Einzeldaten errechnet wurde und nicht wie in Abschnitt 6.1 durch die Mittelwertbildung über die bereits auf Ebene der Raumordnungsregionen aggregierten Daten, unterscheiden sich diese beiden Werte voneinander $(12,29$ zu 12,49).
} 


\section{Abbildung 6: Humankapitalindex von Ost- und Westdeutschland für unter 35Jährige im Vergleich}

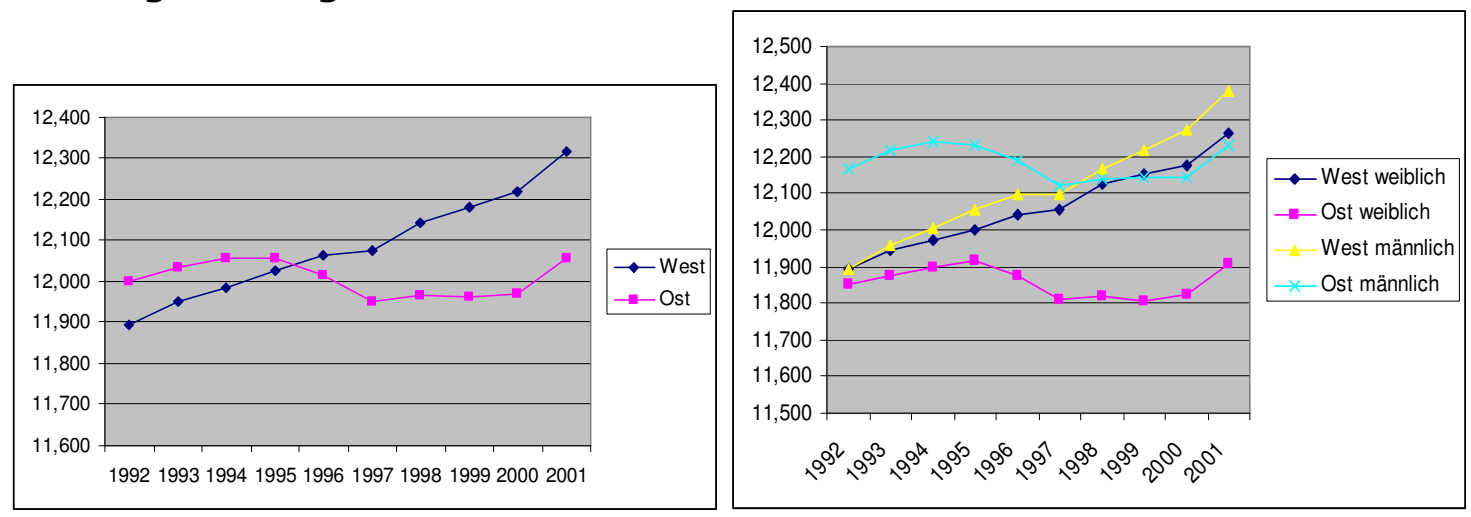

Das Ergebnis verdeutlicht aber auch, dass der Bildungsstand alleine - ohne günstige Rahmenbedingungen - kein Impuls für Beschäftigung ist. Wäre dies der Fall, so müsste Ostdeutschland weniger von Arbeitslosigkeit betroffen sein als Westdeutschland. Mit diesen Daten nicht zu widerlegen ist die Annahme der Wachstumstheorie, die nicht von Niveauunterschieden ausgeht, sondern von zeitlichen Veränderungsraten.

\subsection{Regionale Alterszusammensetzung der Beschäftigten ${ }^{8}$}

Um die dritte Hypothese zu prüfen, soll ein altersstandardisierter Humankapitalindex errechnet werden, aus dem hervorgeht, ob für die Alterszusammensetzung von Beschäftigten eine regionale Disparität in seinem Niveau und seiner Entwicklung zu beobachten ist.

Für das Jahr 2001 zunächst wurden die Mittelwerte der Schuljahre je Alterklasse in den westdeutschen Regionen berechnet (für jedes Geburtsjahr). Diese wurden dann der Gesamtdatei zugespielt und für jede Region wiederum ein Mittelwert durch Aggregation errechnet. Auf diese Weise enthält man einen Index, der widerspiegelt, wie hoch der Humankapitalindex wäre, wenn dieser einzig durch die Altersstruktur der Beschäftigten bestimmt wäre.

Im Ergebnis sind die Unterschiede sehr viel geringer sind als bei der Verwendung des ursprünglichen Humankapitalindex: von 12,21 Jahren (Emsland) bis 12,47 (Region München), der Unterschied beträgt also nur 0,26 Jahre und somit etwa 2,13\% ${ }^{9}$ bei einem Gesamtdivergenz von $12,8 \%$ des Humankapitalindexes. Dies bedeutet, dass sich die Regionen in ihrer Humankapitalausstattung nicht wesentlich aufgrund des Alters ihrer beschäftigten Bevölkerung im Bildungswesen unterscheiden.

Diese Tatsache hat sich seit 1975 nur unwesentlich verändert: 0,98\% beträgt der Unterschied zwischen dem niedrigsten und dem höchsten alterstypischen Humankapitalindex bei einer Gesamtdivergenz von 8,6\% des Humankapitalindexes. Hier liegen die Werte zwischen 11,93 und 11,14 Jahren. Für einzelne Regionen ist jedoch eine Veränderung zu verzeichnen: Die Differenz des alterstypischen Indexes von 1975 zu 2001 beträgt zwischen $9,13 \%$ und $17,05 \%$.

\footnotetext{
${ }^{8}$ Um den alterstypischen Index zu berechnen, wurden zunächst die Fälle herausgefiltert, für die keine Angaben gemacht werden können, da entweder die Bildungsvariable oder die Altersangaben nicht vorhanden ist.

${ }^{9}$ Berechnung: Diff (höchster - tiefster Wert) / tiefsten Wert * 100 .
} 


\section{Abbildung 7: Humankapitalindex und seine Differenz zum alterstypischen Index 2001}

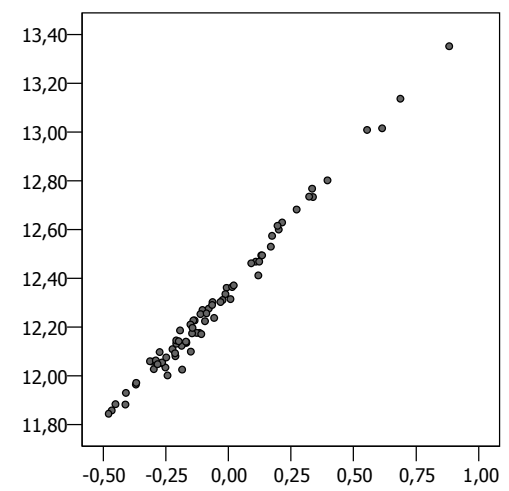

Es wurde mit den Originalwerten des Humankapitalindexes, ohne Berücksichtigung des Alters, eine Differenz der beiden Werte je Region berechnet. Für 2001 liegt diese Differenz zwischen -.48 Jahren (Region "Landshut") und +.88 Jahren (Region „München"), für 1975 zwischen -.50 (Region "Schwarzwald-Baar-Heuberg") und +.38 (Region "Hamburg"). Diese Werte spiegeln wegen der geringeren regionalen Unterschiede die Struktur des ursprünglichen Humankapitalindexes wider. Die Unterschiede zwischen den Indices sind größer als die altersbedingten regionalen Humankapitalunterschiede. Dies kann graphisch gut im Streudiagramm dargestellt werden (siehe Abbildung 7), in dem auf der x-Achse die Differenz vom alterstypischen zum ursprünglichen Index aufgetragen ist und auf der $y$ Achse der ursprüngliche Humankapitalindex. Die Linie verläuft linear.

\subsection{Weitere Bildungsvariablen}

Empirisch wie theoretisch besteht keine Einigkeit über die Erträge von spezifischer und allgemeiner Bildung. Verschiedene weitere Bildungsvariablen wurden daher untersucht, die beide Kategorien umfassen.

Es wurde vermutet, dass bei unsicherer Kausalität die Berufsbildung und der Verbleib der so Ausgebildeten in der Region in Zusammenhang mit der Beschäftigungssituation steht. Daher wurden die sich in Ausbildung befindlichen Personen anteilig an den Beschäftigten berechnet.

Eine andere Vermutung bezog sich auf die Rolle des Abiturs. Es wurden daher auch die Anteile der Abiturienten berechnet, die entweder direkt vom Arbeitsmarkt aufgenommen wurden und die eine Ausbildung unternommen haben. Dieser Anteil wurde nur unter der jungen Bevölkerung (bis 35 Jahre) berechnet, um somit zu erfassen, ob junge Leute nach dem Abitur in den Regionen auf dem Arbeitsmarkt nachgefragt werden.

Außerdem wurde noch eine Variable hinzugenommen, die häufig als Indikator für Humankapital im Sinne eines innovations- und produktivitätsstützenden Faktors verwendet wird: der Anteil von Studierten. Da in Deutschland eine Besonderheit in der Unterscheidung von Fachhochschulen und Universitäten vorliegt, wurden zwei Indikatoren unterschieden: Studierte Beschäftigte an Universitäten und studierte Beschäftigte mit Universitäts- oder Fachhochschulabschluss.

Wie die verschiedenen Bildungsvariablen zusammenhängen, kann der folgenden Tabelle entnommen werden. 


\section{Tabelle 3: Korrelationskoeffizienten zwischen den verschiedenen Bildungsvariablen}

\begin{tabular}{|c|c|c|c|c|}
\hline 2001 & $\begin{array}{l}\text { Abiturienten- } \\
\text { anteil }\end{array}$ & $\begin{array}{l}\text { Auszubilden- } \\
\text { denanteil }\end{array}$ & $\begin{array}{l}\text { Anteil Studierte } \\
\text { (Uni) }\end{array}$ & $\begin{array}{l}\text { Anteil aller } \\
\text { Studierten }\end{array}$ \\
\hline Humankapitalindex & $.917^{* *}$ & $-.602 * *$ & $.938^{* *}$ & $.942 * *$ \\
\hline Abiturientenanteil & & $-.448^{* *}$ & $.885^{* *}$ & $.844 * *$ \\
\hline Auszubildendenanteil & & & $-.610 * *$ & $-.665^{*}$ \\
\hline Anteil Studierte (Uni) & & & & $.975 * *$ \\
\hline \multicolumn{5}{|l|}{1975} \\
\hline Humankapitalindex & $.521 * *$ & $-.236 *$ & $.682 * *$ & $.747 * *$ \\
\hline Abiturientenanteil & & $-.441 * *$ & $.719 * *$ & $.749 * *$ \\
\hline Auszubildendenanteil & & & $-.440 * *$ & $-.498 * *$ \\
\hline Anteil Studierte (Uni) & & & & $.948^{* *}$ \\
\hline
\end{tabular}

* Die Korrelation ist auf dem 5\%-Niveau signifikant.

** Die Korrelation ist auf dem 1\%-Niveau signifikant.

Die verschiedenen Bildungsvariablen sind alle positiv und hoch miteinander korreliert. Eine Ausnahme bildet der Auszubildendenanteil, der negativ mit den anderen Variablen korreliert. Diese Variable könnte insofern ein Indikator dafür sein, dass junge Menschen in diesen Regionen die Ausbildung dem Abitur und dem Studium vorziehen.

\subsection{Der Zusammenhang von Bildung und Beschäftigung}

Wenngleich letztlich erst in einer Wachstumsgleichung, die eine Reihe verschiedener Faktoren enthält, die bekanntermaßen zu berücksichtigen sind, der Einfluss der Bildungsvariablen auf das Beschäftigungswachstum untersucht werden kann, so soll dennoch in einem ersten Schritt auf den einfachen Zusammenhang eingegangen werden.

Es wird das Beschäftigungswachstum der sozialversicherungspflichtig Beschäftigten betrachtet. Diese können mit der Regionalstichprobe berechnet werden oder von den Berechnungen des BBR ausgegangen werden. Die selbst berechnete Variable und die Variable des BBR korrelieren nahezu perfekt miteinander. In den 74 westdeutschen Regionen beträgt der Korrelationskoeffizient $r=.939$ für das Wachstum in den Jahren 1995 bis 2001; für das Wachstum in den Jahren 1990 bis 2000 ist $r=.956$. Diese Entwicklungen können aus beiden Datensätzen gewonnen werden; neuere Entwicklungen sind für die Regionalstichprobe nicht möglich, da diese Daten bis 2001 enthält; ältere Daten liegen für die INKAR-Daten nicht vor. Unterschiede ergeben sich vor allem dadurch, dass in den eigenen Berechnungen nur solche Beschäftigte berücksichtigt wurden, für die Bildungsangaben vorliegen oder durch das gewählte Imputationsverfahren logisch solche hergeleitet wurden. Spätere Untersuchungen zeigen jedoch, dass die Differenz nicht zu bedeutend anderen Ergebnissen führt.

Die regionalen Beschäftigungszuwächse sind in den vergangen Jahren allerdings eher gering und in vielen Fällen auch negativ. So ist der Mittelwert für die Entwicklung von 1995 bis 2001 in der Regionalstichprobe - 0,54\% für alle Regionen und 2,3\% für die westdeutschen Regionen. Seit 1975, in der langfristigen Perspektive, beträgt das Wachstum im Durchschnitt 30,41\%.

Das Histogramm in Abbildung 9 zeigt die Verteilung des Beschäftigungswachstums in beiden Zeiträumen. 


\section{Abbildung 8: Verteilung des Beschäftigungswachstums für 74 westdeutsche Raumordnungsregionen für 1995-2001 und 1975-2001}

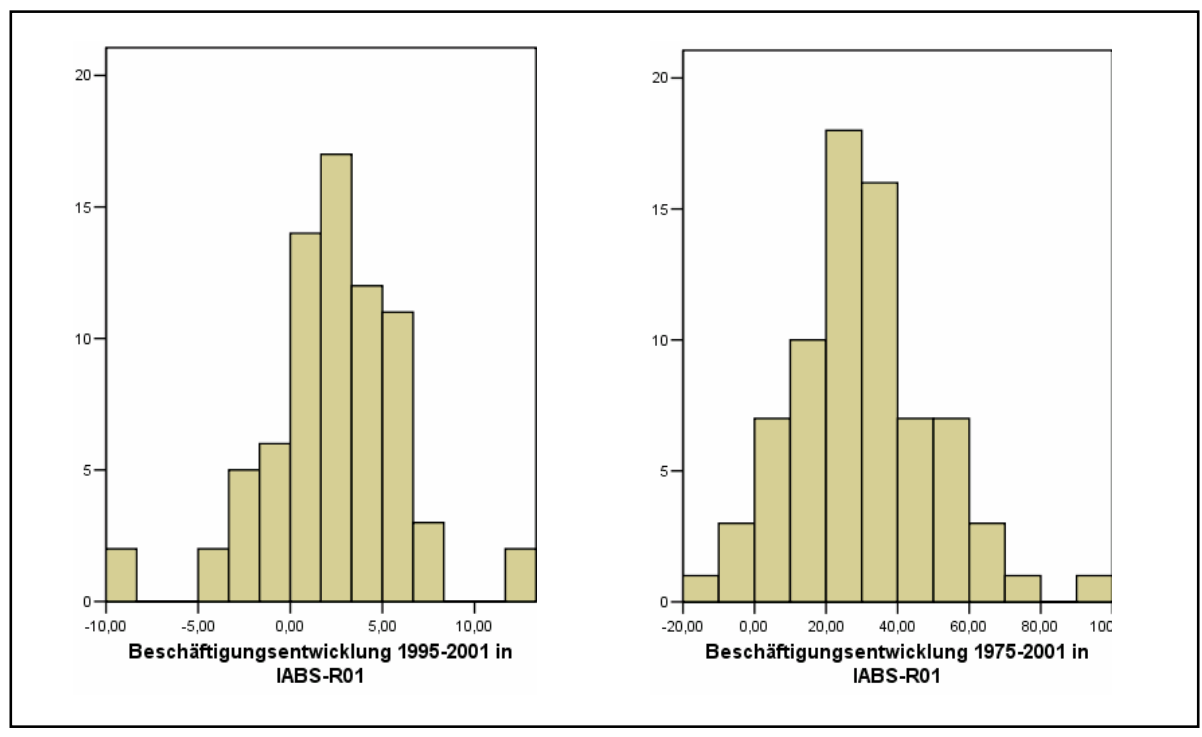

Die Ausgangssituation im Hinblick auf die verschiedenen Bildungsvariablen im Jahr 1975 und das Wachstum ist nur mit dem Anteil der Auszubildenden an allen Beschäftigten signifikant korreliert. Auch die Veränderung des Humankapitalindexes ist mit dem Beschäftigungswachstum unkorreliert.

Dies gilt nicht gleichermaßen in der Situation der vergangenen Jahre. Für die Entwicklung über den Zeitraum von 1995 bis 2001 findet sich mit vielen Bildungsvariablen eine signifikante Korrelation, außer mit dem Anteil der Abiturienten und dem Anteil der Auszubildenden an den Beschäftigten. Die meisten Korrelationen sind jedoch negativ, sowohl im Hinblick auf das Niveau der Bildungsvariable im Jahr 1995 wie auf deren Veränderung. Dies würde - ohne die Berücksichtigung weiterer Variablen - bedeuten, dass sich eine Höherqualifizierung der Beschäftigten negativ auf das Wachstum auswirken würde. Die einzige positive Variable, die gleichzeitig die höchste Korrelation mit dem Beschäftigungswachstum aufweist, ist die Entwicklung des Humankapitalindexes im gleichen Zeitraum ( $r=.541)$.

Die einfachen bivariaten Korrelationen sind nicht eindeutig. Diese Ergebnisse sprechen für die Anwendung einer Wachstumsgleichung, wie sie in den Arbeiten von Mankiw et al. (1992) und vielen Folgeuntersuchungen gerechnet wurden. Das Wachstum der Beschäftigung scheint am ehesten - und wie es scheint: bestenfalls - mit dem Wachstum der Bildungsvariablen einherzugehen.

Die Tatsache, dass die sich der Anteil der in Ausbildung befindlichen Personen im Jahr 1975 mit dem langfristigen Beschäftigungswachstum zusammenhängt, lässt mehrere Interpretationen zu. So könnte es ein Hinweis auf die beginnende Bildungsexpansion sein, die zum Beschäftigungswachstum beitrug oder einfach parallel zum Wirtschaftsboom statt fand. Die anderen Bildungsvariablen messen mehr als die anderen Variablen den bereits erreichten Bildungsstand. Andererseits hat auch die Veränderung des Humankapitalindexes in der gleichen Zeit keinen signifikanten Zusammenhang aufgezeigt. Der Zusammenhang könnte auch auf die Bedeutung und den Bedarf von Ausbildungsberufen zu dieser Zeit hindeuten. Da keine Daten zu Arbeitslosigkeit oder sonstigeren relevanten Daten auf der Ebene der Raumordnungsregionen vor 1990 vom BBR vorliegen, kann dieser Tatbestand jedoch nicht tiefer gehend analysiert werden. 


\subsection{Der Einfluss von Branchen}

Branchen- und Industriemix werden in regionalökonomischen Studien häufig bedacht und als Erklärung für das wirtschaftliches und Produktivitätswachstum herangezogen. Welchen Zusammenhang Branchen und ihre Interaktion mit dem Humankapital für das Beschäftigungswachstum haben, wird anhand der Daten der Regionalstichprobe überprüft. Im Scientific Use File des Datensatzes wird zwischen 16 Branchen unterschieden.

Um den Brancheneinfluss auf die Veränderung des Humankapitalindexes berechnen zu können, wird zunächst eine Aggregation der Einzeldaten vorgenommen, die einen Humankapitalindex pro Branche und Jahr ausgibt. Die tiefere Aggregation je Raumordnungsregion wurde aufgegeben, da in vielen Fällen die Fallzahl zu gering ist.

Es zeigt sich, dass durch die Wiedervereinigung alle Branchen einen Sprung gemacht haben hin zu einem höheren Indexwert, was die Sonderstellung Ostdeutschlands einmal mehr bestätigt. Die gleiche Berechnung wurde anschließend noch einmal ohne Ostdeutschland durchgeführt (siehe Abbildung 9).

\section{Abbildung 9: Branchenverläufe 1975-2001 für das in ihnen enthaltene Humankapital ohne Ostdeutschland}

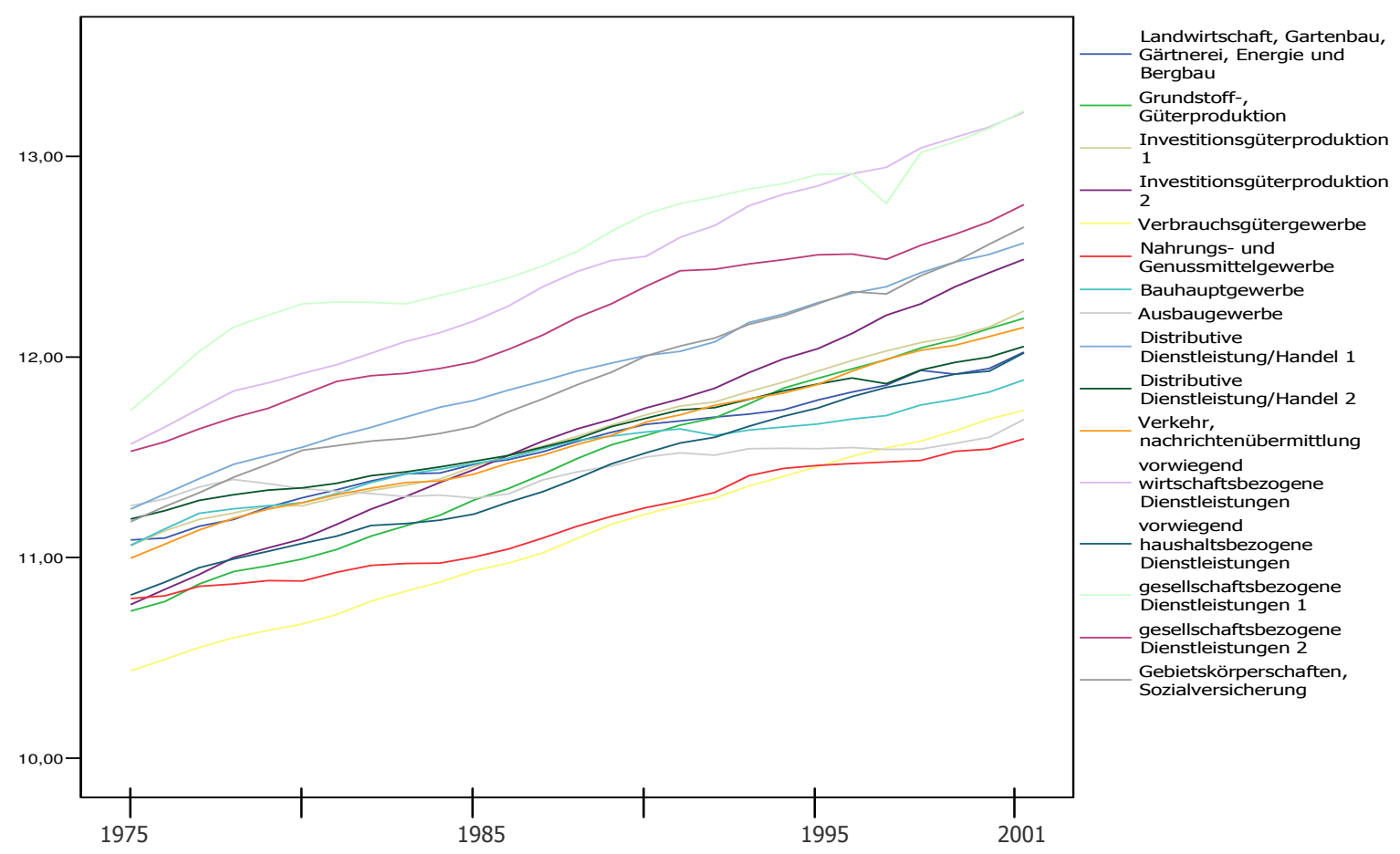

In einigen Branchen werden Personen mit einem höheren Bildungsabschluss vergleichweise mehr beschäftigt als in anderen. Diese humankapitalintensiven Branchen sind erwartungsgemäß die Dienstleistungsbranchen (ausgenommen sind hier die haushaltsbezogenen Dienstleistungen). Wenig humankapitalintensiv sind besonders das Nahrungs- und Genussmittelgewerbe, das Verbrauchsgütergewerbe und das (Aus-) Baugewerbe. 
Auffällig im Zeitverlauf sind einige Schwankungen oder Stagnationen zwischen den Jahren 1996 und 1998 in etwa der Hälfte der Branchen. Äußerst stark ist diese bei den "gesellschaftsbezogenen Dienstleistungen 1, z. B. Heime, Krankenhäuser". ${ }^{10}$

Insgesamt ist eine Aufwärtstendenz seit 1975 zu beobachten, was mit der allgemeinen Steigerung des Indexes übereinstimmt. Seit einigen Jahren tritt schließlich in vielen Branchen eine Stagnation ein, die zum Teil bereits Anfang der 90er Jahre beginnt. Diese fällt besonders bei den ohnehin wenig humankapitalintensiven Branchen "Bauhauptgewerbe", „Ausbaugewerbe" und "Nahrungs-/ Genussmittelgewerbe" auf. Die größten Steigerungen sieht man vor allem bei den "vorwiegend wirtschaftsbezogenen Dienstleistungen".

Mit Daten zu aggregierten Zahlen zu Beschäftigten ${ }^{11}$ in verschiedenen Sektoren kann die Bedeutung der Dienstleistungsbranchen unterstrichen werden. In Abbildung 10 sind die Anteile der Beschäftigten in Dienstleistungsberufen in den verschiedenen Raumordnungsregionen auf der $x$-Achse abgetragen. In Abbildung 11 ist ein noch deutlicherer, negativer Zusammenhang zu erkennen, der zwischen dem Anteil der Beschäftigten in Fertigungsberufen und der Humankapitalintensität zu beobachten ist. Diese beiden Variablen korrelieren für das Jahr 2001 mit einem Koeffizienten von $r=-.843$ miteinander, die Korrelation mit den anteilig in Dienstleistungsberufen Beschäftigten beträgt $r=.757$. Der Humankapitalindex steht demnach stark mit den Berufen in Zusammenhang, die in verschiedenen Branchen einer unterschiedlichen Nachfrage unterliegen.

\section{Abbildungen 10 und 11: Humankapitalindex und Beschäftigte in Dienstleistungsberufen (links) und Fertigungsberufen (rechts), ohne Ostdeutschland}
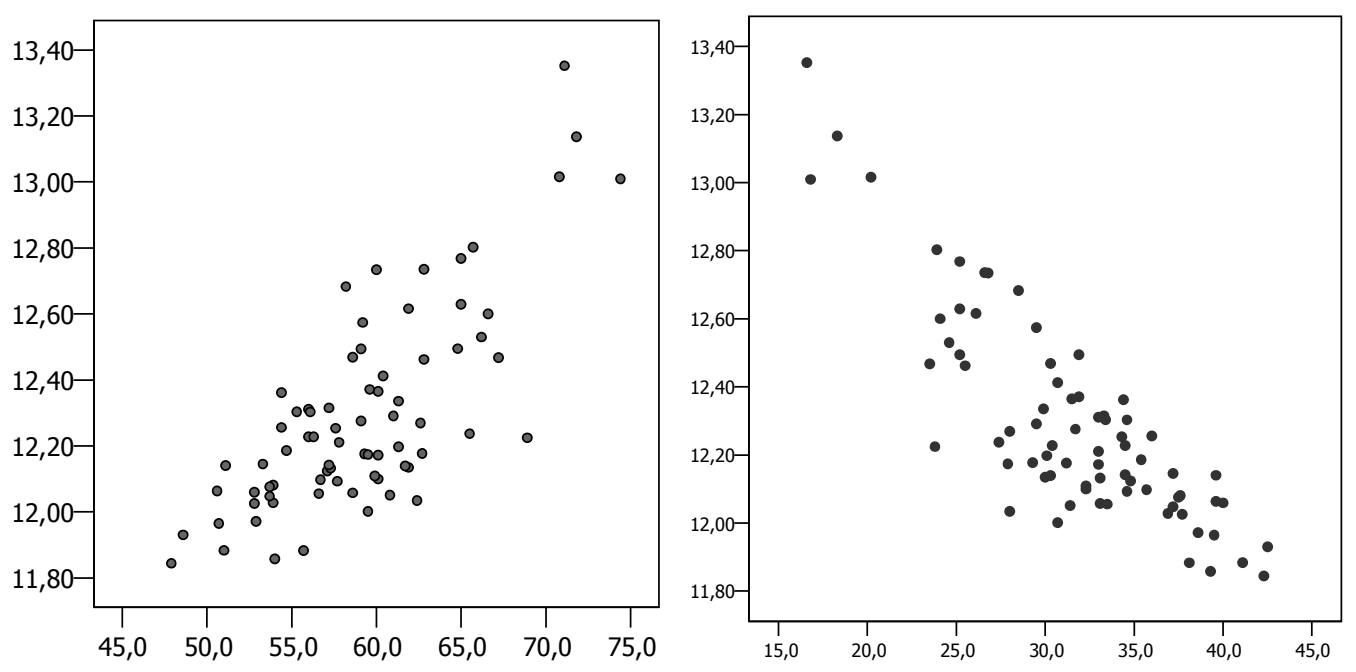

In einem weiteren Schritt wurde der Anteil der Personen, die in den 16 Branchen beschäftigt sind, im Zeitverlauf dargestellt (siehe Abbildung 12). Dazu wurde die Anzahl der Beschäftigten je Wirtschaftssektor und Jahr aus der Regionalstichprobe aggregiert. In Westdeutschland ist besonders der deutliche Anstieg der "vorwiegend wirtschaftsbezogenen Dienstleistungen" in der Absorption von Beschäftigten ersichtlich.

${ }^{10}$ In einer vorhergehenden Berechnung, die auch geringfügig Beschäftigte einschloss, fanden sich noch stärkere Schwankungen, welche zum Jahr 1999 vorliegen, die mit der Aufnahme der geringfügig Beschäftigten in das amtliche Meldeverfahren zu begründen sind. Der Humankapitalindex sinkt in dieser Betrachtung.

${ }^{11}$ Hierzu wurden Daten aus der INKAR-Datei verwendet 
Auch bei den "gesellschaftsbezogenen Dienstleistungen" ist ein Anstieg gut sichtbar. Stetig sinkende Beschäftigtenzahlen befinden sich dahingegen in den Bereichen Grundstoff/Güterproduktion und Verbrauchsgütergewerbe. In einigen Branchen sinkt die Anzahl der Personen nach Beginn der 90er Jahre deutlich. Dies betrifft unter anderem die "Investitionsgüterproduktion" und „Bauhauptgewerbe". Insgesamt 6 Branchen haben im Jahr 2001 im Vergleich zu 1975 weniger sozialversicherungspflichtig Beschäftigte aufzuweisen. Die Dienstleistungsbranchen (ohne haushaltsbezogene Branchen) haben den höchsten Zuwachs mit jeweils über 100\%.

Abbildung 12:

Verlauf der Beschäftigtenzahlen je Wirtschaftszweig ohne Ostdeutschland

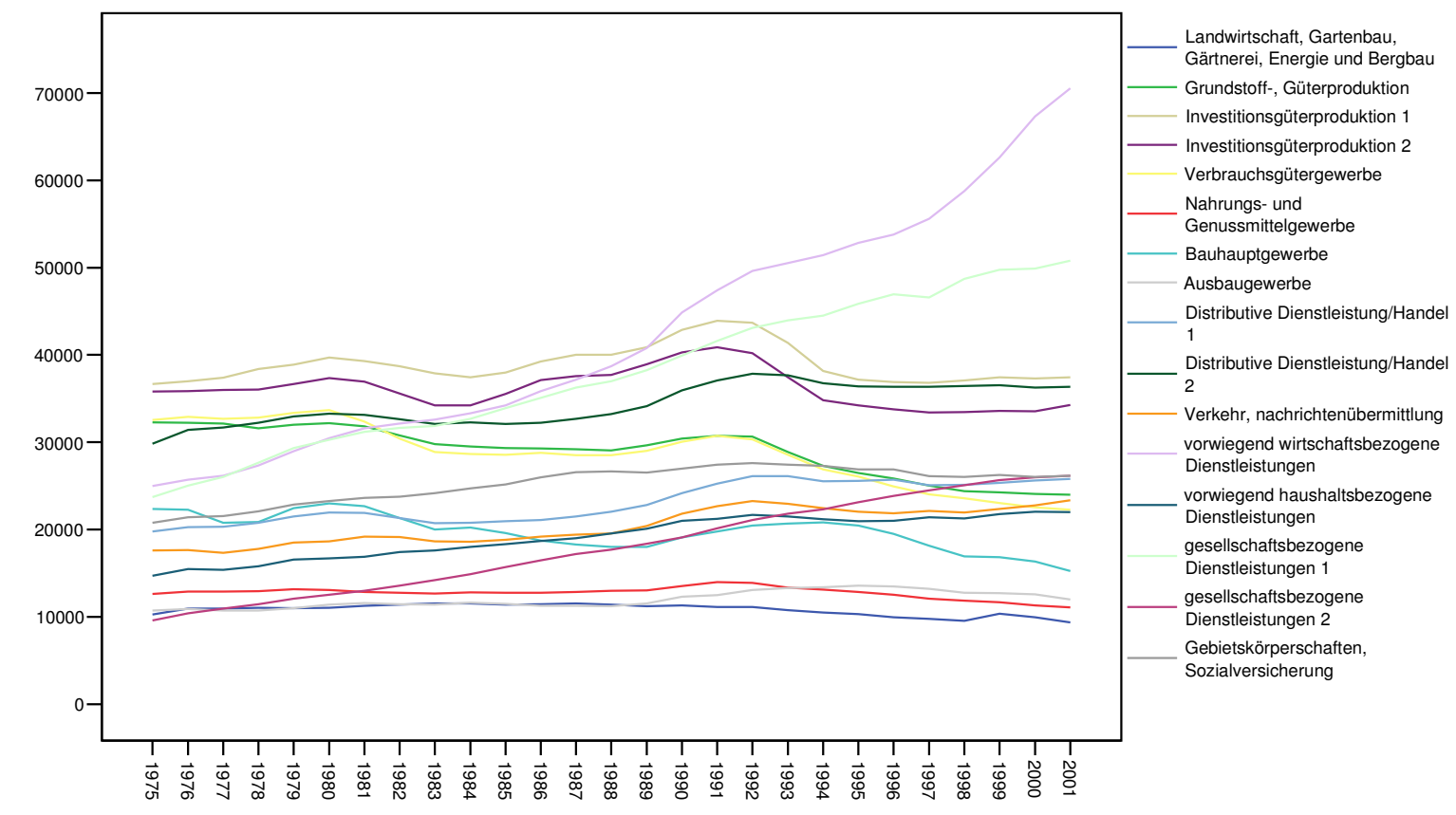

Die nächste Frage ist, wie die branchentypischen von den tatsächlichen Werten bezüglich des Humankapitals abweichen. Ein branchentypischer Wert zeigt dabei an, wie humankapitalintensiv sich eine Region darstellen würde, wenn allein die Branchenanteile entsprechend des Bundesschnitts die Humankapitalintensität bestimmten. Hierfür wurde den im Datensatz vertretenen Personen der durchschnittliche Wert des Humankapitalindexes für die jeweilige Branche in Westdeutschland zugewiesen und je Raumordnungsregion aggregiert. Der Korrelationskoeffizient mit diesem branchentypischen Index liegt nahe bei Null für alle untersuchten Jahre ${ }^{12}$.

Differiert der branchentypische Wert vom tatsächlichen Wert, so sind diese Werte von den Branchen unabhängig. Dieser Differenzwert weist jedoch nur einen geringen direkten Zusammenhang mit der Beschäftigungsentwicklung auf, der nur in wenigen Fällen auf dem 5\%-Niveau signifikant ist.

Dieses Ergebnis zeigt an, dass die Branchenanteile an sich keine Erklärung für die Beschäftigungsentwicklung ist. Über die Branchen hinaus wirken jedoch andere Faktoren. Eine hochsignifikante Korrelation zwischen beiden Indices mit den Beschäftigten in Dienstleistungsberufen (positiv; z.B. für das Jahr $2001 \mathrm{r}=.726$ ) und in Fertigungsberufen (negativ; z.B. für das Jahr $2001 \mathrm{r}=-.814$ ) spricht dafür, dass die Beschäftigung sich dort

12 Es handelt sich um die Jahre 1975/1990/1995/2001 (branchentypischer Index) und die Beschäftigungsentwicklung für die Jahre 1975-2001, 1990-1998/2000 und 1995-2001. 
stark entwickelt, wo innerhalb der Branchen humankapitalintensive Berufe ausgeübt werden und vermutlich andere Bereiche in der Wertschöpfungskette berührt werden als in anderen Regionen. Für die Entwicklung der Beschäftigten in Dienstleistungsberufen gilt jedoch gleichermaßen, dass nur eine eher schwache Korrelation zu verzeichnen ist, die nicht in allen Fällen signifikant ist.

In der langfristigen Perspektive, über die der branchentypische und der branchenunabhängige Index keinen Aufschluss geben können, ist ein anderer Indikator aussagekräftiger. Die Branchenzusammensetzung von 1975 wurde hierzu fortgeschrieben und der Zusammensetzung im Jahr 2001 gegenübergestellt, indem die sich hypothetisch hieraus ergebende Beschäftigtenzahl berechnet wurde. Eine Differenz der hypothetischen und der aktuellen Beschäftigtenzahlen im Jahr 2001 wurde errechnet. Diese steht in einem stark signifikanten Zusammenhang mit dem regionalen Humankapitalindex ( $r=-.749$ für den Humankapitalindex von 1975). Dies lässt den Schluss zu, dass eine (positive) Branchenentwicklung durch das Humankapital begünstigt wird.

Für den Zusammenhang von Branchenzusammensetzung, Humankapitalintensität und Beschäftigung kann aus den beschriebenen Ergebnissen festgehalten werden, dass in allen Branchen die Humankapitalintensität gestiegen ist, wenngleich sie in den Dienstleistungen besonders hoch ist. Humankapitalintensive Dienstleistungsberufe werden innerhalb der verschiedenen Branchen in den Raumordnungsregionen in unterschiedlichem Maße angeboten. Der branchenunabhängige Humankapitalindex steht mit dem Beschäftigungswachstum in einem signifikanten Zusammenhang. Die Humankapitalintensität im Ausgangsjahr wirkt sich auch langfristig auf die Branchenzusammensetzung aus. Die Kausalität ist in diesen Fällen jedoch unklar.

\subsection{Erklärung des Beschäftigungswachstums}

Die bisherigen Darstellungen zum Zusammenhang der Bildungsvariablen und Beschäftigungswachstum sind vor allem deskriptiver Natur oder zeigen einfache Zusammenhänge ohne die Berücksichtigung weiterer erklärender Variablen auf. Daher sollen im folgenden Abschnitt weitere Faktoren einbezogen werden, die in der empirischen Analyse von Wachstum typischerweise bedacht werden. Dies wird anhand der Beschäftigungsentwicklung für 1995 bis 2001 geschehen, für die Daten in INKAR und IABSR01 vorliegen.

Hierzu soll zunächst eine einfache KQ-Regression geschätzt werden, in der das Beschäftigungswachstum als abhängige Variable eingeht und die Veränderung der Humankapitalvariable als unabhängige Variable. Darüber hinaus wird als erklärende Variable die Arbeitslosigkeit zu Beginn der beobachteten Periode einbezogen, da dies die Ausgangssituation darstellt, von der aus ein Konvergenzprozess stattfinden kann. In einem weiteren Schritt werden zusätzlich zu diesen beiden Variablen die Investitionen in Sachkapital (R3) und eine Stadt-Land-Dummy (R4) berücksichtigt. Die Ergebnisse sind in Tabelle 4 dargestellt. 
Tabelle 4: Ergebnisse der KQ-Regression

\begin{tabular}{|l|c|c|c|}
\hline Variablen/Regressionen & \multicolumn{2}{|c|}{ R1 } & \multicolumn{2}{|c|}{ R2 } & R3 \\
\hline $\begin{array}{c}\text { Gesamtmodell } \\
\text { Abh. Variable: } \\
\text { Beschäftigungswachstum }\end{array}$ & $\boldsymbol{r}^{\mathbf{2}=\mathbf{6 7 9}}$ & $\boldsymbol{r}^{\mathbf{2}=.682}$ & $\boldsymbol{r}^{\mathbf{2}=. \mathbf{7 1 2}}$ \\
\cline { 2 - 5 } & $-.642^{* *}(.000)$ & $-.639^{* *}(.000)$ & $-.661^{* *}(.000)$ \\
\hline Arbeitslosigkeit (1995) & $.292^{* *}(.000)$ & $.278^{* *}(.000)$ & $.308^{* *}(.000)$ \\
\hline $\begin{array}{l}\text { Humankapitalindex } \\
(1995-2001)\end{array}$ & & $.051(.405)$ & $.088(.147)$ \\
\hline Investitionen (1995-2001) & & & $.186^{* *}(.003)$ \\
\hline Stadt-Land-Dummy & & &
\end{tabular}

** Signifikant auf dem 1\%-Niveau

Die Arbeitslosigkeit im Ausgangsjahr geht mit negativem Vorzeichen in die Regression ein und hat in allen Varianten den höchsten Erklärungsanteil. Dies deutet darauf hin, dass keine regionale Konvergenz vorliegt. Der Humankapitalindex ist ebenfalls in allen Varianten hochsignifikant und in diesem Fall positiv.

In internationalen Studien wird jedoch häufig ein erweitertes Solow-Modell verwendet, das der Wachstumstheorie entstammt. Wachstumsregressionen nach dem Solow-Modell enthalten wie zuvor die Investitionen in Sachkapital, die Bildungsvariable und außerdem eine Variable, die sich aus dem Bevölkerungswachstum, dem technologischen Wandel (üblicherweise mit " $\rho$ " bezeichnet) und der Amortisationsrate des Sach- und Humankapitals

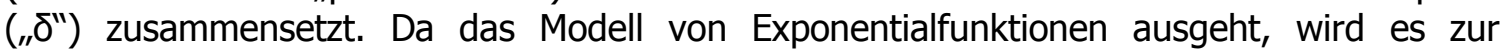
Schätzung durch die Anwendung von Logarithmen linearisiert. Es wird mit stetigen Wachstumsraten gerechnet. Für den hier beobachteten Zeitraum stellt sich dies wie folgt dar:

$$
\begin{gathered}
\ln \left(B_{2001} / B_{1995}\right)=\alpha+\beta_{0} \ln \left(A_{L Q} Q_{1995}\right)+\beta_{1} \ln \left(\operatorname{INV}_{2001} \operatorname{INV}_{1995}\right)+\beta 2 \ln (n+\rho+\delta)+\beta_{3} \\
\ln \left(B_{L} D_{2001 /} \operatorname{BILD~}_{1995}\right)+\varepsilon
\end{gathered}
$$

wobei B die Beschäftigung ist, ALQ die Arbeitslosigkeit, INV die Investitionen, $\mathrm{n}$ das Bevölkerungswachstum und $(n+\rho+\delta)$ die zusammengesetzte Variable, für die üblicherweise angenommen wird, dass $\rho+\delta$ dem Wert 0,05 entspricht, und BILD die Bildungsvariable meint. Für die Investitionsvariable wurde die auf Ebene der Raumordnungsregionen vorliegende Variable „Investitionen im produzierenden Gewerbe" herangezogen. ${ }^{13}$ Für die im Zeitverlauf betrachteten Variablen werden wieder durchschnittliche jährliche Werte herangezogen. Um die Ausgangssituation darzustellen, wurde die Arbeitslosigkeit als Variable gewählt. Eine weitere Möglichkeit besteht in der Hinzunahme der Beschäftigungsquote im Jahr 1995. Die Veränderung der beiden Variablen korreliert mit $r=-.816$ für die Jahre 1995-2001, jedoch ist ihr Zusammenhang ohne Berücksichtigung ihrer Veränderung weniger eindeutig (beispielsweise findet sich für 1998 und 1999 keine Signifikanz). Die Arbeitslosigkeit ist der Beschäftigungsquote prinzipiell vorzuziehen, da die Beschäftigtenquote sich auf die Einwohnerzahl bezieht, nicht aber auf die erwerbsfähige Bevölkerung. Die Ergebnisse sind in Tabelle 5 dargestellt.

${ }^{13}$ Mit den Daten der EU auf Ebene der NUTS-Einteilung wurde die Investitionsentwicklung im produzierenden Gewerbe mit denen anderer Branchen verglichen. Die Korrelation der Variablen untereinander betrug jeweils 1 . 


\section{Tabelle 5: Ergebnisse der Wachstumsregressionen (Beschäftigung)}

\begin{tabular}{|c|c|c|c|}
\hline \multirow{2}{*}{$\begin{array}{l}\text { Variable } \\
\text { Gesamtmodell }\end{array}$} & \multicolumn{3}{|c|}{$\begin{array}{c}\text { Stand. } \beta \text { - Koeffizient } \\
\text { (Signifikanzniveau) }\end{array}$} \\
\hline & $r^{2}=.435$ & $r^{2}=.440$ & $r^{2}=.373$ \\
\hline Arbeitslosigkeit 1995 & $\begin{array}{l}-.095 \\
(.406)\end{array}$ & $\begin{array}{l}-.196 \\
(.112)\end{array}$ & $-.067(.575)$ \\
\hline $\begin{array}{l}\text { Investitionen (1995- } \\
\text { 2001) }\end{array}$ & $\begin{array}{l}.120 \\
(.204)\end{array}$ & $\begin{array}{l}.117 \\
(.214)\end{array}$ & $.120 \quad(.233)$ \\
\hline $\begin{array}{l}\text { Interaktionsterm mit } \\
\text { Bevölkerungswachstum } \\
(n+\rho+\delta)\end{array}$ & $\begin{array}{l}.541^{* *} \\
(.000)\end{array}$ & $\begin{array}{l}.504 * * \\
(.000)\end{array}$ & $\begin{array}{l}.532^{* *} \\
(.000)\end{array}$ \\
\hline Humankapitalindex & $\begin{array}{l}.253^{* *} \\
(.008)\end{array}$ & & \\
\hline Abiturientenanteil & & $\begin{array}{l}.285^{* *} \\
(.006)\end{array}$ & \\
\hline Studiertenanteil & & & $-.027 \quad(.784)$ \\
\hline
\end{tabular}

Es zeigt sich, dass die Regionen bezüglich ihrer Arbeitslosenquote nicht konvergieren, denn diese Variable ist nicht signifikant, ebenso wie die Investitionen, welche auf Beschäftigung keine Auswirkungen zu haben scheinen. Das Bevölkerungswachstum wiederum ist in allen Modellen einflussreich und zwar mit positivem Vorzeichen. Das ist ein Hinweis auf die Bedeutung des Arbeitskräfteangebots, das auch durch die Zuzüge von Arbeitskräften aus anderen Regionen zusammenhängt. Das Wachstum des Humankapitalindexes und des Abiturientenanteils erklären das Beschäftigungswachstum ebenfalls hochsignifikant. Die Signifikanz des Abiturientenanteils bestätigt somit die anfangs erwähnten Vermutungen US-amerikanischer Untersuchungen.

Der Anteil studierter Beschäftigter ist nicht signifikant. Ein ähnliches Ergebnis zeigt sich bei Bade (2001), der den Anteil von in Forschung und Entwicklung beschäftigter Personen untersuchte. Er wies diesbezüglich auf die Notwendigkeit räumlicher Nähe von Forschung und Entwicklung sowohl zu Fertigung wie zu Verdichtungsräumen hin. Ein Teil dieses Ergebnisses könnte damit erklärt werden.

Die Wachstumregressionen ermöglichen zwar Erkenntnisse über die Beschäftigungsentwicklung, die Investitionen spielen hier jedoch in keinem Fall eine Rolle. Wird statt der Beschäftigung das Wachstum des Pro-Kopf-Einkommens als abhängige Variable untersucht, erweisen sich die Investitionen als bedeutsam, nicht aber der Interaktionsterm, der das Bevölkerungswachstum einschließt. Diese Unterschiede spiegeln strukturelle Unterschiede zwischen den Mechanismen von Beschäftigungs- und Wirtschaftswachstum wider. Die einzige signifikante Bildungsvariable ist der Humankapitalindex, der die durchschnittlich absolvierten Jahre formaler Bildung ausdrückt (Tabelle 6). 
Tabelle 6: Ergebnisse der Wachstumsregressionen (Pro-Kopf-Einkommen)

\begin{tabular}{|c|c|c|c|}
\hline \multirow{3}{*}{$\begin{array}{l}\text { Variable } \\
\text { Gesamtmodell }\end{array}$} & \multicolumn{3}{|c|}{$\begin{array}{c}\text { Stand. } \beta \text { - Koeffizient } \\
\text { (Signifikanzniveau) }\end{array}$} \\
\hline & & & \\
\hline & $r^{2}=.787$ & $r^{2}=.723$ & $r^{2}=.728$ \\
\hline $\begin{array}{l}\text { Pro-Kopf-Einkommen } \\
1995\end{array}$ & $-.894 * * \quad(.000)$ & $-.849 * *(.000)$ & $\begin{array}{c}-.785^{* *} \\
(.000)\end{array}$ \\
\hline $\begin{array}{l}\text { Investitionen (1995- } \\
\text { 2001) }\end{array}$ & $-.144 *(.014)$ & $.148^{*} \quad(.026)$ & $\begin{array}{l}.174 * * \\
(.010) \\
\end{array}$ \\
\hline$(n+\rho+\delta)$ & $-.002(.976)$ & $.014 \quad(.857)$ & $.029 \quad(.709)$ \\
\hline Humankapitalindex & $.270 * * \quad(.000)$ & & \\
\hline Abiturientenanteil & & $.108(.138)$ & \\
\hline Studierendenanteil & & & $.076 \quad(.240)$ \\
\hline
\end{tabular}

Für das Wachstum der Beschäftigung wie des Bruttoinlandsprodukts erweist sich somit das Humankapital als bedeutend. Dies gilt in beiden Fällen für den Humankapitalindex, für den Abiturientenanteil findet sich ein Zusammenhang lediglich mit dem Beschäftigungswachstum.

\section{Schlussfolgerungen}

Die Ergebnisse dieser Untersuchung sollte mit Daten der IAB-Regionalstichprobe Zusammenhänge zwischen Bildung und Beschäftigung auf regionaler Ebene in Westdeutschland aufzeigen. Es wurden aus der Wachstums- und Regionalforschung die Hypothesen abgeleitet, dass Konvergenz zwischen den Regionen im Bezug auf die Humankapitalintensität nicht vorliegt, dass die Altersstruktur der Beschäftigten keine Rolle spielt und das ein Zusammenhang zwischen Bildung und Beschäftigung nachweisbar ist.

Die Ergebnisse zeigen, dass eine Konvergenz des Humankapitalindexes nicht vorliegt. Dies gilt auch für die Disparitäten zwischen männlichen und weiblichen Humankapitalindices, die zwar innerhalb der Regionen konvergieren, nicht aber zwischen den Regionen. Insgesamt ist der Humankapitalindex in allen Regionen und Branchen gestiegen.

Das Humankapital ist so verteilt, dass viele ländliche Gebiete insbesondere im Süden Deutschlands relativ wenig humankapitalintensiv sind. Dennoch sind die Wachstumsraten der Beschäftigung in diesen Regionen nicht zwingend bedenklich, was entsprechend der Theorie in der Regionalforschung mit innovationsförderlichen Wirkungen von Agglomerationsräumen erklärt werden kann.

Ein alterstypischer Humankapitalindex macht deutlich, dass die regionalen Humankapitalunterschiede nicht auf die Alterszusammensetzung der Beschäftigten zurückzuführen sind. Gleiches konnte für einen branchentypischen Index gefunden werden. Einen indirekten Brancheneffekt gibt es dennoch, denn die langfristige Branchenentwicklung ist günstiger für Regionen, die humankapitalintensiv sind. Eine Analyse von Daten mit detaillierteren Brancheninformationen könnten hier in späteren Untersuchungen Aufschluss geben.

Die Wachstumsregressionen zeigen, dass der Humankapitalindex mit Blick auf das Beschäftigungswachstum nicht eindeutig als positiv und bedeutend bestätigt werden kann. Erkenntnisse aus der Regionalforschung weisen darauf hin, dass dies zum Teil mit der räumlichen Nähe wenig humankapitalintensiver Regionen zu Verdichtungszentren in Zusammenhang stehen könnte, die überdurchschnittliche Wachstumsraten verzeichnen. 
Andererseits sind regionale Spillover-Effekte für Beschäftigung weniger weitreichend als für den Produktivitätszuwachs (Niebuhr, 2000). Die Jahre formaler Bildung und der Anteil an Beschäftigten mit Abitur sind - im Gegensatz zum Anteil von Studierten an den Beschäftigten - in den Wachstumsregression signifikant. Es kann auch bestätigt werden, dass der Einfluss von Humankapital auf die Beschäftigung weniger eindeutig ist als auf das wirtschaftliche Wachstum. Allerdings konnten die Wachstumsregressionen aufgrund der Verfügbarkeit von Daten auf der Ebene von Raumordnungsregionen bisher nur der kurze Zeitraum von 1995 bis 2001 berücksichtigt werden. Wünschenswert wäre eine langfristigere Perspektive.

\section{Literaturverzeichnis}

BADE, F.-J. (2001): "Regionale Entwicklungstendenzen und Unterschiede des Humankapitals," in Ordnungspolitik als konstruktive Antwort auf wirtschaftspolitische Herausforderungen. Festschrift zum 65. Geburtstag von Paul Klemmer, H.-F. Eckey, D. Hecht, M. Junkerheinrich, H. Karl, N. Werbeck\&R. Wink (Hrsg.). Stuttgart: Lucius \& Lucius, 337-364.

BARRO, R. J.\&LEE, J.-W. (1993): "International comparisons of educational attainment," Journal of Monetary Economics, 32, 363-394.

- (2000): International data on educational attainment: updates and implications. Center for International Development at Harvard University. Working Paper No. 42.

BATEY, P. (2002): "Human resources and regional development," in Regional development reconsidered, G. Atalik\&M. M. Fischer (Hrsg.). Berlin: Springer, 53-75.

BECKER, G. (1993): "Human Capital: A theoretical and empirical analysis with special reference to education," University of Chicago Press.

BENSI, M. T., BLACK, D. C.\&Dow, M. R. (2004): "The education-growth relationship: Evidence from Real State Panel Data," Contemporary Economic Policy, 22, 281-298.

BERTOCCHI, G.\&SPAGAT, M. (2003): "The evolution of modern educational systems. Technical versus general education, distributional conflict, and growth," Journal of Development Economics, 73, 559-582.

BLIEN, U., SüDEKUM, J.\&WOLF, K. (2006): "Local employment growth in West Germany: a dynamic panel approach," Labour Economics, 13, 445-458.

BRÄUNINGER, M.\&NiEBUHR, A. (2005): Agglomeration, spatial interaction and convergence in the EU. Hamburg. HWWA Discussion Paper 322.

BRUNELLI, G., LUPI, C.\&ORDINE, P. (2001): "Widening differences in Italian regional unemployment," Labour Economics, 8, 103-129.

BUTTLER, F.\&TESSARING, M. (1993): "Humankapital als Standortfaktor Mitteilungen aus der Arbeitsmarkt- und Berufsforschung, 26, 467-476.

Coulombe, S., TREMBLAY, J.-F.\&MARChAND, S. (2004): Literacy scores, human capital and growth across fourteen OECD countries. Statistics Canada. Catalogue no. 89-552, no. 11.

DE LA FUENTE, A.\&DOMÉNECH, R. (2000): Human capital in growth regressions: How much difference does data quality make? , OECD Economics Department. . OECD Economics Department Working Papers, 262. 
ECKEY, H.-F., KOSFELD, R.\&TÜRCK, M. (2005): "Regionale Produktionsfunktionen mit Spillover-Effekten für Deutschland - Empirischer Befund und wirtschaftspolitische Implikationen," Schmollers Jahrbuch, 125, 239-267.

ELHORST, J. P. (2003): "The mystery of regional unemployment differentials: theoretical and empirical explanations," Journal of Economic Surveys, 17, 709-748.

FARHAUER, O.\&GRANATO, N. (2006): "Regionale Arbeitsmärkte in Westdeutschland: Standortfaktoren und Branchenmix entscheidend für Beschäftigung.," IAB-Kurzbericht Nr. 04/2006, http://doku.iab.de/kurzber/2006/kb0406.pdf.

FESER, E. J. (2003): "What regions do rather than make: A proposed set of knowledge-based occupation clusters," Urban Studies, 40, 1937-1958.

FitZenBERger, B., Osikominu, A.\&VÖLTER, R. (2005): Imputation rules to improve the education variable in the IAB Employment Subsample. Mannheim. ZEW Discussion Paper No. 05-10.

Galiani, S., Lamarche, C., Porto, A.\&SosA-Escudero, W. (2005): "Persistence and regional disparities in unemployment (Argentina 1980-1997)," Regional Science and Urban Economics 35, 375 - 394.

"Geppert, K., Gornig, M.\&WerWATZ, A. (2005): "Entwicklung gegen den Trend räumlicher Konvergenz - Empirische Ergebnisse zum Erklärungsbeitrag sektorspezifischer Entwicklungen," http://www.tagung05.unibonn.de/Papers/Gornig.pdf: Verein für Socialpolitik: Jahrestagung 2005.

Goldin, C.\&KATZ, L. F. (2001): "The legacy of U.S. educational leadership: Notes on distribution and economic growth in the 20th century," American Economic Review, 91, 18-23.

IWAHASHI, R. (2007): "A theoretical assessment of regional development effects on the demand for general education," Economics of Education Review, forthcoming.

JONES, G.\&SCHNEIDER, W. J. (2006): "Intelligence, human capital, and economic growth: A Bayesian Averaging of Classical Estimates (BACE) approach," Journal of Economic Growth, 11, 71-93.

LEVINE, R.\&RENELT, D. (1991): "A sensitivity analysis of cross-country growth regressions," American Economic Review, 82, 942-963.

MANKIW, N. G., ROMER, D.\&WEIL, D. N. (1992): "A contribution to the empirics of economic growth," Quarterly Journal of Economics, 107, 407-432.

NiebuHR, A. (2000): Räumliche Wachstumszusammenhänge - empirische Befunde für Deutschland. Hamburg. HWWA Discussion Paper 84.

OSTERMEIER, M.\&BLOSSFELD, H.-P. (1998): "Wohneigentum und Ehescheidung. Eine Längsschnittanalyse über den Einfluß gekauften und geerbten Wohneigentums auf den Prozeß der Ehescheidung," Zeitschrift für Bevölkerungswissenschaft, 23, 39-54.

Pugno, M. (2005): "The service paradox and endogenous economic growth," Structural Change and Economic Dynamics, 17, 99-115.

RAMAKRISHNAN, U.\&CERISOLA, M. (2004): Regional economic disparities in Australia. IMF Working Paper. WP/04/144.

SinN, H.-W. (2001): "Sozialisierung des Humankapitals," Welt am Sonntag, 8. April, 56.

WALSH, P. P. (2003): "The cyclical pattern of regional unemployment flows in Poland," Economic Systems, 27, 155-169. 
WEBER, A. A. (1998): "Humankapital, Schulbildung und Wirtschaftswachstum: Eine kritische Betrachtung der Literatur," in Bildung und WIrtschaftswachtum, R. K. von Weizsäcker (Hrsg.). Berlin: Duncker \& Humblot, 49-76. 